\title{
THE APPLICATION OF PHYTOPLANKTON IN ECOLOGICAL ASSESSMENT OF THE BALKHASH LAKE (KAZAKHSTAN)
}

\author{
BARINOVA, S. $.^{*}-$ KRUPA, E. ${ }^{2}-$ TSOY $\mathrm{V}^{3}-$ PONAMAREVA, L. ${ }^{3}$ \\ ${ }^{1}$ Institute of Evolution, University of Haifa \\ Mount Carmel, 199 Abba Khoushi Ave., Haifa 3498838, Israel \\ (phone: +972-4-824-9697, +972-4-824-9799; fax: +972-4-824-6554) \\ ${ }^{2}$ Republican State Enterprise on the Right of Economic Use "Institute of Zoology", Ministry of \\ Education and Science, Science Committee, Almaty 050060, Republic of Kazakhstan \\ (e-mail: elena_krupa@mail.ru) \\ ${ }^{3}$ Kazakh Research Institute of Fishery LLP \\ Zheltoksan street 20, Balkhash town100300, Republic of Kazakhstan \\ (e-mail: assylbekova@mail.ru) \\ *Corresponding author \\ e-mail: sophia@evo.haifa.ac.il \\ (Received $10^{\text {th }}$ Jan 2018; accepted $26^{\text {th }}$ Mar 2018)
}

\begin{abstract}
The Balkhash Lake is a unique natural water body with a gradient of the total content of dissolved salts along its longitudinal axis and the increased level of toxic pollutions. To assess the spatial distribution of environmental factors along the Balkhash Lake surface, methods of bioindication and statistical mapping were used. An assessment of the overall level of toxic pollution was carried out using the Water Ecosystem State Index (WESI). Phytoplankton has been used to assess the ecological state of the Balkhash Lake in the summer of 2004. A total of 92 species were found in the algal communities, with prevalence of Chlorophyta in the western, fresher water part, and Bacillariophyta in the eastern, brackish part. Mapping showed a positive relationship between the species richness in algal Divisions and macrophytes. Changes in the proportion of indicator species within the composition of phytoplankton reflected the spatial gradient of depth, TDS, $\mathrm{pH}$-values, organic pollution. According to bioindication data, the waters of the Balkhash Lake are moderately polluted, water quality class III. The WESI index calculation revealed the effect of the total toxic pollution on the phytoplankton of the Western Balkhash. The spatial distribution of the WESI index showed that in the zone the influence of river flow has stimulating effect of biogenic elements on phytoplankton but was partially leveled by the increased level of toxic pollution.
\end{abstract}

Keywords: bioindication, heavy metals, organic pollution, total dissolved solids, WESI index, phytoplankton, Balkhash Lake, Kazakhstan

\section{Introduction}

The Balkhash Lake is located in arid zone of the southeastern Kazakhstan, on sedimentary carbonate soils with granites, porphyries, tuff and slates in its northern and western parts (Sidorenko, 1972; Leonov, 2013). The water area is the third largest following the Caspian Sea and Aral Sea. One distinct feature about the lake is its spatial heterogeneity of the content of Total Dissolved Solids (TDS) in water (Tarasov, 1961). The arid climate also conditions interannual changes of TDS associated with fluctuations of level of the Balkhash Lake.

Due to the rich reserves of polymetallic ores (Mazurov, 2005), there are mining and processing industry facilities in the region (Kudekov, 2002). The territory south of Balkhash Lake is used for agriculture and pasture cattle. The combination of natural 
and anthropogenic factors determines the increased level of pollution and substantial heterogeneity of ecological conditions on various parts of the water area of the Balkhash Lake.

Recently, methods of the biological analysis have been used more and more for assessment of quality of water. Phytoplankton serves as a good instrument for that purpose (Barinova et al., 2006; Barinova and Chekryzheva, 2014; Klymiuk and Barinova, 2016; Krupa et al., 2016; Krupa and Barinova, 2016; Barinova and Krupa, 2017), and its application has not exhausted its potential (Barinova et al., 2015a). Water quality assessment is carried out both on the basis of structural indicators of biological communities (abundance, biomass, ratio of divisions in quantitative indicators, average cell volume, diversity indices), and with indicator species distribution.

The basis for using indicator algae for bioindication is their relation to environmental factors - the amount of organic matter, oxygen conditions, salinity of water, etc. The system for assessing the level of organic pollution of water bodies on the basis of the ratio of indicator species of various systematic groups was developed by V. Sládeček (1973). S. S. Barinova (Barinova et al., 2006; Barinova, 2011; Barinova and Fahima, 2017; Barinova, 2017a) compiled the electronic database on algal indicators with species-specific ecological preferences in the world. This database on the ratio of algal species, in addition to the level of organic contamination, allows assessing a wider range of environmental factors such as temperature, oxygen conditions, salinity, prevailing depths, and water $\mathrm{pH}$.

When assessing the ecological status of any waterbody, it is most difficult to detect the effect of toxic pollution, in particular the effect of heavy metals, on biological communities. This is due to the fact that the toxicity of heavy metals depends on the multidirectional action of a complex of environmental factors. So, high temperature intensifies, and increased salinity and $\mathrm{pH}$ reduce the toxic properties of heavy metals for living organisms ( $\mathrm{Li}$ et al., 2013). These same environmental factors affect the absorption of heavy metals by submerged plants (Fritioff et al., 2005) and thereby to self-purification processes in aquatic ecosystems.

One of the new methods, allowing in an integrated form to assess the effect of the general level of toxic pollution on phytoplankton communities, is the Water Ecosystem State Index (WESI) (Barinova et al., 2006; Barinova, 2011, 2017a, b). The indicator significance of this index was demonstrated by us earlier when assessing the ecological state of water bodies in Kazakhstan and other regions (Barinova et al., 2011, 2015a, b; Barinova and Chekryzheva, 2014; Barinova and Krupa, 2017).

The research on phytoplankton of Balkhash Lake has begun in 1929. In the years 1929-1930 the microalgae were represented by 308 species and forms (Abrosov, 1973). The main contribution to the diversity of algal flora was made by benthic species of algae, which did not play a significant role in plankton. Data on quantitative parameters of algal flora during this period are absent. The Kapshagay reservoir began to fill from the Ili River in 1970 and the Ili River is the main filling source of the Balkhash Lake. Reduction of the volume of river flow led to a decrease in the water level and intensification of toxic pollution of the lake. The biomass of phytoplankton in the summer of 1976-1978 after the completion of the Kapshagay reservoir filling, reached 0.51-1.64 $\mathrm{g} \mathrm{m}^{-3}$ (Vorobyeva et al., 1982; Fokina, 1982). During the period from 1998 to 2012, the variety of planktonic algae ranged from 82 to 146 species (Ponomareva et al., 2005; Krupa et al., 2014a). The mean annual biomass averaged $0.94 \mathrm{~g} \mathrm{~m}^{-3}$, fluctuating from 0.17 to $1.86 \mathrm{~g} \mathrm{~m}^{-3}$ across different years. The diatom algae dominated the biomass 
most often, followed by blue-greens. Studies of long-term dynamics (1972-2012) revealed the influence of the invader, the bivalve Monodacna colorata on phytoplankton biomass (Krupa et al., 2013), and the relationship between the long-term variability of phytoplankton biomass and regional and global climatic factors (Krupa et al., 2014b). The spatial distribution of total species richness (Barinova et al., 2017) and structural variables of phytoplankton (Krupa et al., 2017a, 2018) was studied along the Balkhash Lake in a gradient of abiotic factors based on the materials of 2004.

There is no information in the recent publications on the indicator species of planktonic algae and their using for assessment of organic and toxic pollution level of the Balkhash Lake.

The aim of the present work is to assess the organic contamination, hydrochemical and oxygen conditions, and water $\mathrm{pH}$ by the mapping of spatial distribution of indicator species of planktonic algae in the Balkhash Lake as well as assess the overall level of toxic pollution of the reservoir on the basis of spatial distribution of the Water Ecosystem State Index (WESI). For Lake Balkhash this will be done for the first time.

\section{Materials and methods}

\section{Sampling and chemical analysis}

The research of phytoplankton, the content of biogenic elements and heavy metals in the water was carried out by means of a grid of 58 stations (Figs. 1 and 2) in June and July of 2004. The measures of the temperature and $\mathrm{pH}$ values of the surface water layers were taken in the field environment. Water transparency was measured with Secchi disk. Coordinate referencing of the stations was done by Garmin eTrex GPS-navigator. The samples for heavy metals were fixed in the site by adding nitric acid; samples for biogenes were fixed with chloroform. All collected samples were transported to the lab in an icebox. One-liter samples of phytoplankton were sampled from surface layer of the lake water.

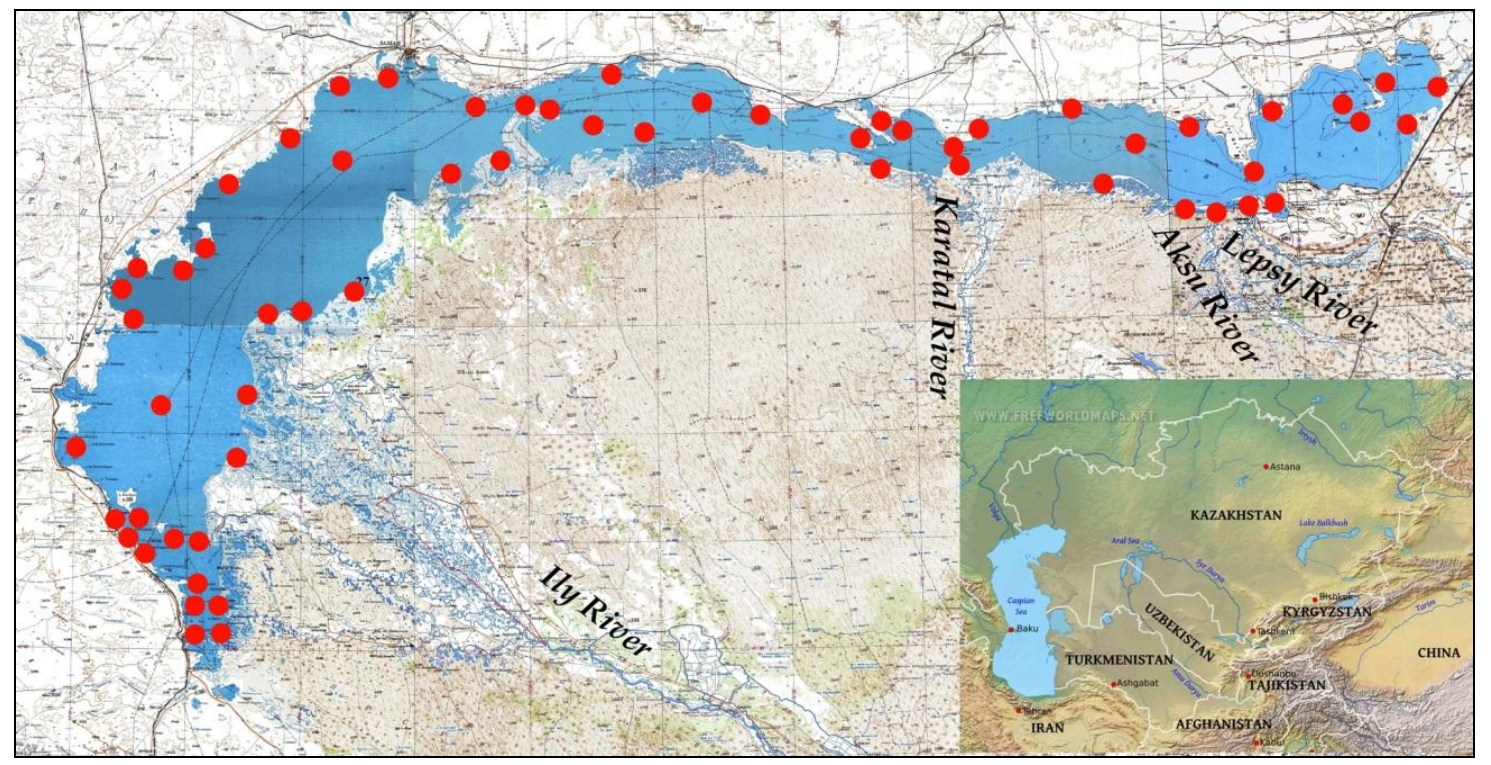

Figure 1. Map of sampling stations on the Balkhash Lake, 2004 


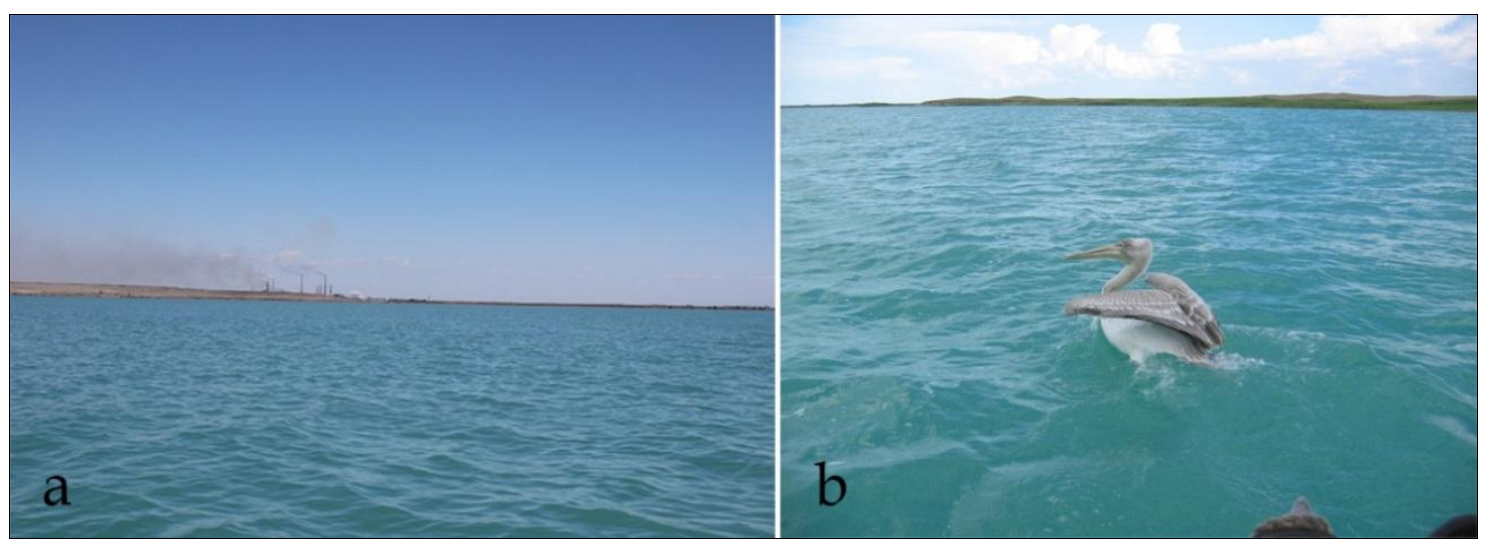

Figure 2. Western (a) and eastern parts (b) of the Balkhash Lake, 2004

Conventional methods of chemical analysis of water were used (Semenova, 1977; Fomin, 1995). Water samples were analyzed in three - four replications. The error of estimate for major ions in the water was $0.5-5.0 \%$, depending on the analysis. Concentrations of heavy metals were determined by the atomic absorption method using an AAS-1N spectrophotometer (Germany). The device allows for the detection of the various chemical elements in complex matrices, including those in the sea and grey water and in the biological objects in micro-trace quantities Test-sensitivity of AAS-1N spectrophotometer $-0.001-0.0025 \%$ Mass.

The settling method was used (Kiselev, 1956) to process phytoplankton samples. Species identification of planktonic algae was performed using determinant handbooks for relevant divisions (Gollerbach et al., 1953; Zabelina et al., 1951; Popova, 1955; Moshkova et al., 1986; Palamar-Mordvintseva, 1982; Matvienko, 1954).

\section{Bioindication}

The ecological characteristics of algal species were obtained from the database compiled for freshwater algae of the world from multiple analyses of algal biodiversity by Barinova et al. (2006), according to substrate preference, temperature, oxygenation, $\mathrm{pH}$, salinity, organic enrichments, as well as $\mathrm{N}$-uptake metabolism and trophic states according Van Dam et al. (1994). The ecological groups were separately assessed according to their significance for bioindications. Species that respond predictably to environmental conditions were used as bioindicators for particular variables of aquatic ecosystems, the dynamics of which are related to environmental changes.

The correspondence between the classificational indicators of ecosystem according to the biogenic elements and those of saprobity indices was conducted through calculating the index of the ecosystem state (WESI, Water Ecosystem State Index) as the quotient from the classification rank on biota divided by the classification rank on the nitrates. The WESI index ranges from 0 to 9 . If it is less than one, then the ecosystem is exposed to the toxic effects; if equal to or greater than 1 - the selfpurification is not suppressed (Barinova et al., 2006, Barinova, 2011).

\section{Statistical methods}

The statistical methods used were those recommended by Heywood (2004) for the development of taxonomic studies. The innovative approach with spatial mapping in 
wafer plots construction was applied using the Statistica 12.0 program for analysis of biological and environmental variables distribution over the lake surface. For this type of calculation we used GIS coordinates of each sampling station. Data of biological variables for the Balkhash lake sampling stations were inserted to the Statistica 12.0 program and maps were created for each variable. Statistically generated maps are better if we have numerous sampling points. The maps have some prognostic properties and let to assume the level of each mapped variable between sampling points.

\section{Description of the study site}

The Uzun-Aral Channel divides the Balkhash Lake into two parts: western, which is shallow and wide (Western Balkhash), and eastern, which is narrow and deep (Eastern Balkhash). The lake is elongated from southwest to northeast (Fig. 1). Its length makes up to $614 \mathrm{~km}$, and the area is approximately $16400 \mathrm{~km}^{2}$ if the water level is $342 \mathrm{~m}$ above sea level. Width of the lake reaches $9-19 \mathrm{~km}$ in the eastern part and up to $74 \mathrm{~km}$ in western part (Kudekov, 2002). The lake is fed by rivers Ili, Karatal, Lepsy and Aksu. There is constant flow of water from the west to the east (flow of run-off) (Tarasov, 1961). The movement of water masses begins from the mouth of Ili River and flows into the Western Balkhash. Encroaching on the opposite shore, the current is directed to the northeast and reaches the Uzun-Aral Channel. Further flow is caused by a difference of levels between the western and eastern parts as the Karatal, Lepsy and Aksu rivers do not fully compensate the evaporation from a surface of the Eastern Balkhash.

\section{Results}

\section{Hydrophysical characteristics}

The Western and Eastern Balkhash significantly differ in environmental variables (Table 1). The shallow western part of the lake is characterized by lesser transparency and $\mathrm{pH}$-values of water, poorer distribution of stiff macrophytes (reed, cane, cattail), but it is more overgrown with soft macrophytes (pondweed plants, Myriophyllum). Macrophytes developed mainly in the southern and southeast parts of the lake where rivers discharge their waters such as the Ili River in the Western Balkhash, and the Karatal, Aksu and Lepsy rivers in the Eastern Balkhash.

Table 1. Hydrophysical and morphometric characteristics of Balkhash Lake, summer 2004

\begin{tabular}{c|c|c|c}
\hline Variable & Whole Balkhash & Western Balkhash & Eastern Balkhash \\
\hline Temperature, ${ }^{\circ} \mathrm{C}$ & $24.14 \pm 0.14$ & $23.49 \pm 0.16$ & $24.77 \pm 0.14$ \\
\hline Depth, m & $5.58 \pm 0.49$ & $4.34 \pm 0.28$ & $6.86 \pm 0.81$ \\
\hline Transparency, m & $1.13 \pm 0.15$ & $0.53 \pm 0.02$ & $1.74 \pm 0.25$ \\
\hline Macrophytes Hard, \% & $20.05 \pm 3.03$ & $18.03 \pm 3.00$ & $22.14 \pm 5.36$ \\
\hline Macrophytes Soft, \% & $25.18 \pm 2.68$ & $28.28 \pm 3.29$ & $22.00 \pm 4.24$ \\
\hline pH & $8.63 \pm 0.04$ & $8.52 \pm 0.02$ & $8.74 \pm 0.09$ \\
\hline
\end{tabular}

\section{Hydrochemical and toxicological characteristics}

Total Dissolved Solids (TDS) content in the East Balkhash is statistically significantly higher, than in the western part (Table 2). In Western Balkhash, the 
concentration of nitrates, nitrites, total phosphorus, iron, zinc and copper is higher than in the eastern part. Phosphates, easily oxidized organic matter, cadmium, lead, nickel and cobalt were present in the waters of the East Balkhash in large concentration. The content of ions of ammonium and silicon did not differ in the two parts of the lake.

Table 2. Chemical water variables, toxicants, index $S$ and index WESI for the Balkhash Lake, summer 2004

\begin{tabular}{c|c|c|c}
\hline Variable $^{*}$ & Whole Balkhash & Western Balkhash & Eastern Balkhash \\
\hline $\mathrm{TDS}$ & $2286.0 \pm 236.0$ & $1080.3 \pm 83.4$ & $3436.8 \pm 286.7$ \\
\hline $\mathrm{Cl}$ & $473.3 \pm 58.9$ & $170.5 \pm 18.6$ & $762.4 \pm 71.3$ \\
\hline $\mathrm{NH}_{4}$ & $0.102 \pm 0.017$ & $0.103 \pm 0.013$ & $0.102 \pm 0.033$ \\
\hline $\mathrm{NO}_{3}$ & $0.945 \pm 0.244$ & $1.379 \pm 0.410$ & $0.492 \pm 0.226$ \\
\hline $\mathrm{NO}_{2}$ & $0.042 \pm 0.011$ & $0.060 \pm 0.013$ & $0.024 \pm 0.016$ \\
\hline $\mathrm{P}-\mathrm{PO}_{4}$ & $0.017 \pm 0.004$ & $0.011 \pm 0.003$ & $0.023 \pm 0.007$ \\
\hline $\mathrm{P}_{\text {tot }}$ & $0.064 \pm 0.001$ & $0.106 \pm 0.012$ & $0.021 \pm 0.007$ \\
\hline Oxydability & $7.39 \pm 0.46$ & $5.07 \pm 0.40$ & $9.13 \pm 0.45$ \\
\hline $\mathrm{Zn}$ & $0.028 \pm 0.009$ & $0.039 \pm 0.018$ & $0.017 \pm 0.002$ \\
\hline $\mathrm{Cu}$ & $0.018 \pm 0.003$ & $0.022 \pm 0.005$ & $0.013 \pm 0.002$ \\
\hline $\mathrm{Cd}$ & $0.0036 \pm 0.0002$ & $0.0028 \pm 0.0002$ & $0.0044 \pm 0.0002$ \\
\hline $\mathrm{Pb}$ & $0.034 \pm 0.003$ & $0.021 \pm 0.002$ & $0.047 \pm 0.004$ \\
\hline $\mathrm{Ni}$ & $0.039 \pm 0.001$ & $0.037 \pm 0.002$ & $0.042 \pm 0.002$ \\
\hline $\mathrm{Co}$ & $0.013 \pm 0.001$ & $0.010 \pm 0.0005$ & $0.017 \pm 0.001$ \\
\hline $\mathrm{Index} \mathrm{S}$ & $1.85 \pm 0.04$ & $1.94 \pm 0.06$ & $1.78 \pm 0.04$ \\
\hline Index WESI & $1.35 \pm 0.14$ & $0.86 \pm 0.20$ & $1.86 \pm 0.20$
\end{tabular}

*All variables units as $\mathrm{mg} \mathrm{dm}^{-3}$, Oxydability as $\mathrm{mg}_{2} \mathrm{dm}^{-3}$, indices $\mathrm{S}$ and WESI are unitless

\section{Phytoplankton}

Altogether 92 species of phytoplankton were revealed, among them green algae were prevail with 29 species, then follow diatoms (27), cyanobacteria (21), charophytes and euglenophytes (4 each), dinophytes (3), chrysophyte algae (1) (Table 3).

Table 3. Species diversity with ecological preferences in phytoplankton of the Balkhash Lake, summer 2004

\begin{tabular}{l|c|c|c|c|c|c|c|c|c|c|c}
\hline \multicolumn{1}{c|}{ Taxa } & T & Hab & Reo & pH & pH-range & Hal & D & Tro & Aut-Het & Sap & Index s \\
\hline \multicolumn{1}{c|}{ Bacillariophyta } & & & & & & & & & & & \\
\hline Achnanthes sp. & - & - & - & - & - & - & - & - & - & - & - \\
\hline Amphora sp. & - & - & - & - & - & - & - & - & - & - & - \\
\hline $\begin{array}{l}\text { Asterionella formosa } \\
\text { Hassall }\end{array}$ & - & P & st-str & alf & 7.4 & i & sx & o-m & ate & o & 1.35 \\
\hline $\begin{array}{l}\text { Aulacoseira granulata } \\
\text { (Ehrenberg) Simonsen }\end{array}$ & temp & P-B & st-str & ind & $5.8-9.4$ & i & es & me & ate & b & 2.0 \\
\hline \begin{tabular}{l} 
Chaethoceros sp. \\
\hline Cocconeis sp.
\end{tabular} & - & - & - & - & - & - & - & - & - & - & - \\
\hline \begin{tabular}{l} 
Coscinodiscus sp. \\
\hline
\end{tabular} & - & - & - & - & - & - & - & - & - & - & - \\
\hline
\end{tabular}




\begin{tabular}{|c|c|c|c|c|c|c|c|c|c|c|c|}
\hline $\begin{array}{l}\text { Cyclotella meneghiniana } \\
\text { Kützing }\end{array}$ & - & P-B & - & - & - & hl & - & - & - & - & - \\
\hline $\begin{array}{l}\text { Cymbella cymbiformis } \mathrm{C} \text {. } \\
\text { Agardh }\end{array}$ & temp & $\mathrm{B}$ & str & ind & $6.2-9$ & $\mathrm{i}$ & sx & $\mathrm{o}-\mathrm{m}$ & ats & $\mathrm{b}$ & 2.0 \\
\hline Cymbella sp. & - & - & - & - & - & - & - & - & - & - & - \\
\hline $\begin{array}{l}\text { Diatoma hyemalis (Roth) } \\
\text { Heiberg }\end{array}$ & temp & $\mathrm{B}$ & st-str & alf & $6.2-9.0$ & $\mathrm{i}$ & sx & me & ate & $\mathrm{o}-\mathrm{b}$ & 1.5 \\
\hline $\begin{array}{l}\text { Entomoneis paludosa (W. } \\
\text { Smith) Reimer }\end{array}$ & - & P-B & - & alf & - & hl & - & $\mathrm{m}$ & - & b-a & 2.5 \\
\hline Eunothia sp. & - & - & - & - & - & - & - & - & - & - & - \\
\hline $\begin{array}{l}\text { Fragilaria acus (Kützing) } \\
\text { Lange-Bertalot }\end{array}$ & - & $\mathrm{P}$ & st-str & alb & - & $\mathrm{i}$ & es & - & - & $\mathrm{o}-\mathrm{a}$ & 1.8 \\
\hline $\begin{array}{l}\text { Fragilaria crotonensis } \\
\text { Kitton }\end{array}$ & - & $\mathrm{P}$ & st-str & alf & 7.5 & I & es & $\mathrm{m}$ & ate & $o-b$ & 1.5 \\
\hline $\begin{array}{l}\text { Gyrosigma acuminatum } \\
\text { (Kützing) Rabenhorst }\end{array}$ & cool & $\mathrm{B}$ & st-str & alf & - & $\mathrm{i}$ & es & me & ate & $\mathrm{o}-\mathrm{a}$ & 1.95 \\
\hline Melosira sp. & - & - & - & - & - & - & - & - & - & - & - \\
\hline Navicula sp. & - & - & - & - & - & - & - & - & - & - & - \\
\hline $\begin{array}{l}\text { Nitzschia acicularis } \\
\text { (Kützing) W. Smith }\end{array}$ & temp & P-B & & alf & $7.85-8.15$ & $\mathrm{i}$ & es & $\mathrm{e}$ & hce & $\mathrm{a}-\mathrm{o}$ & 2.7 \\
\hline $\begin{array}{l}\text { Nitzschia sigmoidea } \\
\text { (Nitzsch) W. Smith }\end{array}$ & & P-B & st-str & alf & $6.2-8.8$ & $\mathrm{i}$ & & $\mathrm{e}$ & ate & $\mathrm{b}-\mathrm{a}$ & 2.5 \\
\hline Nitzschia sp. & - & - & - & - & - & - & - & - & - & - & - \\
\hline $\begin{array}{l}\text { Rhoicosphenia } \\
\text { abbreviata (C. Agardh) } \\
\text { Lange-Bertalot }\end{array}$ & - & B & st-str & alf & 6.7 & $\mathrm{i}$ & es & me & ate & $\mathrm{o}-\mathrm{a}$ & 1.9 \\
\hline $\begin{array}{l}\text { Rhopalodia gibba } \\
\text { (Ehrenberg) Otto Müller }\end{array}$ & temp & $\mathrm{B}$ & - & alf & $6.2-9.0$ & $\mathrm{i}$ & es & o-m & - & $o-b$ & 1.4 \\
\hline Surirella sp. & - & - & - & - & - & - & - & - & - & - & - \\
\hline Tabellaria sp. & - & - & - & - & - & - & - & - & - & - & - \\
\hline $\begin{array}{l}\text { Tryblionella angustata } \\
\text { W. Smith }\end{array}$ & & P-B & st & alf & 7.7 & $\mathrm{i}$ & sx & $\mathrm{m}$ & ats & $\mathrm{o}-\mathrm{b}$ & 1.5 \\
\hline $\begin{array}{l}\text { Tryblionella hungarica } \\
\text { (Grunow) Frenguelli }\end{array}$ & - & P-B & - & alf & - & $\mathrm{mh}$ & $\mathrm{sp}$ & $\mathrm{e}$ & ate & $\mathrm{a}-\mathrm{o}$ & 2.9 \\
\hline \multicolumn{12}{|l|}{ Charophyta } \\
\hline Cosmarium sp. & - & - & - & - & - & - & - & - & - & - & - \\
\hline Desmidium $\mathrm{sp}$. & - & - & - & - & - & - & - & - & - & - & - \\
\hline Staurastrum sp. & - & - & - & - & - & - & - & - & - & - & - \\
\hline $\begin{array}{l}\text { Staurastrum gracile Ralfs } \\
\text { ex Ralfs }\end{array}$ & - & P-B & st & acf & - & $\mathrm{i}$ & - & $\mathrm{m}$ & - & o & 1.3 \\
\hline \multicolumn{12}{|l|}{ Chlorophyta } \\
\hline Ankistrodesmus sp. & - & - & - & - & - & - & - & - & - & - & - \\
\hline $\begin{array}{l}\text { Binuclearia lauterbornii } \\
\text { (Schmidle) Proschkina- } \\
\text { Lavrenko }\end{array}$ & - & - & - & - & - & - & - & - & - & $\mathrm{o}-\mathrm{a}$ & 1.8 \\
\hline $\begin{array}{l}\text { Botryococcus braunii } \\
\text { Kützing }\end{array}$ & - & P-B & st & ind & - & $\mathrm{i}$ & - & - & - & $\mathrm{o}-\mathrm{b}$ & 1.5 \\
\hline Characiopsis sp. & - & - & - & - & - & - & - & - & - & - & - \\
\hline Characium sp. & - & - & - & - & - & - & - & - & - & - & - \\
\hline
\end{tabular}




\begin{tabular}{|c|c|c|c|c|c|c|c|c|c|c|c|}
\hline Chlamydomonas sp. & - & - & - & - & - & - & - & - & - & - & - \\
\hline Chlorella sp. & - & - & - & - & - & - & - & - & - & - & - \\
\hline Chlorococcum sp. & - & - & - & - & - & - & - & - & - & - & - \\
\hline Cladophora sp. & - & - & - & - & - & - & - & - & - & - & - \\
\hline $\begin{array}{l}\text { Coelastrum microporum } \\
\text { Nägeli }\end{array}$ & - & P-B & st-str & ind & - & $\mathrm{i}$ & - & - & - & $\mathrm{b}$ & 2.3 \\
\hline $\begin{array}{l}\text { Dictyosphaerium simplex } \\
\text { Korshikov }\end{array}$ & - & $\mathrm{P}$ & - & - & - & - & - & - & - & $\mathrm{b}-\mathrm{a}$ & 2.5 \\
\hline Franceia sp. & - & - & - & - & - & - & - & - & - & - & - \\
\hline Lagerheimia sp. & - & - & - & - & - & - & - & - & - & - & - \\
\hline $\begin{array}{l}\text { Monoraphidium minutum } \\
\text { (Nägeli) Komárková- } \\
\text { Legnerová }\end{array}$ & - & P-B & st-str & - & - & $\mathrm{i}$ & - & - & - & $b-a$ & 2.5 \\
\hline $\begin{array}{l}\text { Mucidosphaerium } \\
\text { pulchellum (H.C. Wood) } \\
\text { C. Bock, Proschold \& } \\
\text { Krienitz }\end{array}$ & - & P-B & st-str & ind & - & $\mathrm{i}$ & - & - & - & $\mathrm{b}$ & 2.3 \\
\hline $\begin{array}{l}\text { Oocystis solitaria } \\
\text { Wittrock }\end{array}$ & - & $\mathrm{P}$ & st & ind & - & $\mathrm{i}$ & - & - & - & b-o & 1.7 \\
\hline Oocystis sp. & - & - & - & - & - & - & - & - & - & - & - \\
\hline $\begin{array}{l}\text { Oocystis borgei J.W. } \\
\text { Snow }\end{array}$ & - & P-B & st-str & ind & - & $\mathrm{i}$ & - & - & - & $\mathrm{o}-\mathrm{a}$ & 1.9 \\
\hline Palmella sp. & - & - & - & - & - & - & - & - & - & - & - \\
\hline $\begin{array}{l}\text { Pseudoschroederia } \\
\text { robusta (Korshikov) E. } \\
\text { Hegewald \& E. Schnepf }\end{array}$ & - & P-B & st-str & - & - & $\mathrm{i}$ & - & - & - & $\mathrm{o}-\mathrm{a}$ & 1.9 \\
\hline $\begin{array}{l}\text { Scenedesmus arcuatus } \\
\text { (Lemmermann) } \\
\text { Lemmermann } \\
\end{array}$ & - & P-B & st-str & - & - & $\mathrm{i}$ & - & - & - & $\mathrm{o}-\mathrm{a}$ & 1.9 \\
\hline $\begin{array}{l}\text { Scenedesmus balatonicus } \\
\text { Hortobagyi }\end{array}$ & - & - & - & - & - & - & - & - & - & $\mathrm{b}$ & 2.0 \\
\hline $\begin{array}{l}\text { Scenedesmus } \\
\text { quadricauda (Turpin) } \\
\text { Brébisson }\end{array}$ & - & $\mathrm{P}$ & - & ind & - & $\mathrm{i}$ & - & - & - & $\mathrm{b}$ & 2.1 \\
\hline Selenastrum sp. & - & & & & & & & & & & \\
\hline $\begin{array}{l}\text { Tetradesmus obliquus } \\
\text { (Turpin) M.J. Wynne }\end{array}$ & - & P-B & st-str & ind & - & $\mathrm{i}$ & - & - & - & $\mathrm{b}$ & 2.05 \\
\hline $\begin{array}{l}\text { Tetraëdron caudatum } \\
\text { (Corda) Hansgirg }\end{array}$ & - & P-B & st-str & ind & - & $\mathrm{i}$ & - & - & - & $\mathrm{b}$ & 2.0 \\
\hline $\begin{array}{l}\text { Tetraëdron triangulare } \\
\text { Korshikov }\end{array}$ & - & P-B & st-str & - & - & $\mathrm{i}$ & - & - & - & $\mathrm{b}$ & 2.0 \\
\hline $\begin{array}{l}\text { Tetraëdron minutissimum } \\
\text { Korshikov }\end{array}$ & - & - & - & - & - & - & - & - & - & - & - \\
\hline $\begin{array}{l}\text { Tetrapedia gothica } \\
\text { Reinsch }\end{array}$ & - & - & - & - & - & - & - & - & - & - & - \\
\hline Tetrapedia sp. & - & - & - & - & - & - & - & - & - & - & - \\
\hline \multicolumn{12}{|l|}{ Chrysophyta } \\
\hline Dinobryon sp. & - & - & - & - & - & - & - & - & - & - & - \\
\hline \multicolumn{12}{|l|}{ Cyanobacteria } \\
\hline Anabaena sp. & - & - & - & - & - & - & - & - & - & - & - \\
\hline
\end{tabular}




\begin{tabular}{|c|c|c|c|c|c|c|c|c|c|c|c|}
\hline $\begin{array}{l}\text { Aphanizomenon } \\
\text { flosaquae Ralfs ex Bornet } \\
\text { \& Flahault }\end{array}$ & - & $\mathrm{P}$ & - & - & - & hl & - & $\mathrm{m}$ & - & o-a & 1.95 \\
\hline Aphanothece sp. & - & - & - & - & - & - & - & - & - & - & - \\
\hline $\begin{array}{l}\text { Chroococcus turgidus } \\
\text { (Kützing) Nägeli }\end{array}$ & - & $\begin{array}{c}\mathrm{P}- \\
\mathrm{B}, \mathrm{S}\end{array}$ & aer & alf & - & hl & - & - & - & $x-b$ & 0.8 \\
\hline $\begin{array}{l}\text { Coelosphaerium } \\
\text { kuetzingianum Nägeli }\end{array}$ & - & $\mathrm{P}$ & - & - & - & $\mathrm{i}$ & - & $\mathrm{m}$ & - & $b-o$ & 1.6 \\
\hline \multicolumn{12}{|l|}{$\begin{array}{l}\text { Coelosphaerium pusillum } \\
\text { Van Goor }\end{array}$} \\
\hline Gloeocapsa sp. & - & - & - & - & - & - & - & - & - & - & - \\
\hline $\begin{array}{l}\text { Gloeocapsopsis } \\
\text { crepidinum (Thuret) } \\
\text { Geitler ex Komárek }\end{array}$ & - & B & - & - & - & hl & - & - & - & - & - \\
\hline $\begin{array}{l}\text { Gloeocapsopsis magma } \\
\text { (Brébisson) Komárek \& } \\
\text { Anagnostidis ex Komárek }\end{array}$ & - & $\mathrm{S}$ & - & ind & - & $\mathrm{i}$ & - & - & - & - & - \\
\hline $\begin{array}{l}\text { Gomphosphaeria } \\
\text { aponina Kützing }\end{array}$ & - & P-B & st-str & alf & - & hl & - & o & - & o & 1.2 \\
\hline $\begin{array}{l}\text { Limnococcus limneticus } \\
\text { (Lemmermann) } \\
\text { Komárková, Jezberová, } \\
\text { O. Komárek \& } \\
\text { Zapomelová }\end{array}$ & - & $\mathrm{P}$ & - & - & - & $\mathrm{i}$ & - & $\mathrm{o}-\mathrm{m}$ & - & b-o & 1.65 \\
\hline $\begin{array}{l}\text { Merismopedia glauca } \\
\text { (Ehrenberg) Kützing }\end{array}$ & - & P-B & - & ind & - & $\mathrm{i}$ & - & o-m & - & b-o & 1.75 \\
\hline $\begin{array}{l}\text { Merismopedia smithii De } \\
\text { Toni }\end{array}$ & - & P-B & - & - & - & $\mathrm{i}$ & - & o & - & $o-b$ & 1.5 \\
\hline $\begin{array}{l}\text { Merismopedia tenuissima } \\
\text { Lemmermann }\end{array}$ & - & P-B & - & - & - & hl & - & $\mathrm{e}$ & - & b-a & 2.4 \\
\hline $\begin{array}{l}\text { Merismopedia minima } \mathrm{G} . \\
\text { Beck }\end{array}$ & - & $\mathrm{B}, \mathrm{S}$ & aer & - & - & - & - & o & - & & \\
\hline $\begin{array}{l}\text { Microcystis aeruginosa } \\
\text { (Kützing) Kützing }\end{array}$ & - & $\mathrm{P}$ & - & - & - & hl & - & $\mathrm{e}$ & - & $\mathrm{b}$ & 2.1 \\
\hline $\begin{array}{l}\text { Microcystis pulverea } \\
\text { (H.C. Wood) Forti }\end{array}$ & - & $\begin{array}{c}\mathrm{P}- \\
\mathrm{B}, \mathrm{S}\end{array}$ & - & - & - & $\mathrm{i}$ & - & - & - & $o-b$ & 1.5 \\
\hline Microcystis sp. & - & - & - & - & - & - & - & - & - & - & - \\
\hline Phormodium sp. & - & - & - & - & - & - & - & - & - & - & - \\
\hline $\begin{array}{l}\text { Pseudanabaena } \\
\text { endophytica (Elenkin \& } \\
\text { Hollerbach) Anagnostidis }\end{array}$ & - & Ep & - & - & - & $\mathrm{i}$ & - & - & - & - & - \\
\hline $\begin{array}{l}\text { Snowella lacustris } \\
\text { (Chodat) Komárek \& } \\
\text { Hindák }\end{array}$ & - & $\mathrm{P}$ & - & - & - & $\mathrm{i}$ & - & me & - & b-o & 1.6 \\
\hline Spirulina sp. & - & - & - & - & - & - & - & - & - & - & - \\
\hline \multicolumn{12}{|l|}{ Dinophyta } \\
\hline Gymnodinium sp. & - & - & - & - & - & - & - & - & - & - & - \\
\hline Peridinium sp. & - & - & - & - & - & - & - & - & - & - & - \\
\hline $\begin{array}{l}\text { Prorocentrum cordatum } \\
\text { (Ostenfeld) J.D. Dodge }\end{array}$ & - & $\mathrm{P}$ & - & - & - & $\mathrm{hl}$ & - & - & - & - & - \\
\hline
\end{tabular}




\begin{tabular}{l|c|c|c|c|c|c|c|c|c|c|c}
\hline \multicolumn{1}{c|}{ Euglenophyta } & & & & & & & & & & & \\
\hline Euglena sp. & - & - & - & - & - & - & - & - & - & - & - \\
\hline Phacus sp. & - & - & - & - & - & - & - & - & - & - & - \\
\hline Strombomonas sp. & - & - & - & - & - & - & - & - & - & - & - \\
\hline Trachelomonas sp. & - & - & - & - & - & - & - & - & - & - & - \\
\hline
\end{tabular}

Note: Temperature preferences $(\mathrm{T})$ : temp - temperate temperature, eterm - eurythermic, Substrate preferences (Hab): P - planktonic, P-B - plankto-benthic, B - benthic. Oxygenation and water moving (Reo): st - standing water, str - streaming water, st-str - low streaming water, aer - aerophiles. Acidity $(\mathrm{pH})$ degree according to Hustedt (1957): alf - alkaliphiles, ind - indifferents; acf - acidophiles; alb alkalibiontes. Halobity degree according to Hustedt (1938-1939) (Hal): i - oligohalobes-indifferent, hl halophiles, $\mathrm{mh}$ - mesohalobes. Organic pollution indicators according to Watanabe et al. (1986) (D): sx - saproxenes, es - eurysaprobes, sp - saprophyles. Trophic state (Tro) (Van Dam et al., 1994): o oligotraphentic; o-m - oligo-mesotraphentic; m - mesotraphentic; me - meso-eutraphentic; e eutraphentic; o-e - oligo-eutraphentic. Nitrogen uptake metabolism (Aut-Het) (Van Dam et al., 1994): ats - nitrogen-autotrophic taxa, tolerating very small concentrations of organically bound nitrogen; ate nitrogen-autotrophic taxa, tolerating elevated concentrations of organically bound nitrogen; hne facultatively nitrogen-heterotrophic taxa, needing periodically elevated concentrations of organically bound nitrogen; hce - nitrogen-heterotrophic taxa, needing elevated concentrations of organically bound nitrogen. Species-specific Index of Saprobity (s). Self-purification zone preferences (Sap): $x-$ xenosaprob; o - oligosaprob; o-b - oligo-beta-msosaprob; o-a - oligo-alpha-mesosaprob; b - betamesosaprob; b-o - beta-oligo-mesosaprob; b-a - beta-alpha-mesosaprob; a-o - alpha-oligo-mesosaprob; a - alpha-mesosaprob

In the Western Balkhash, where Chlorophyta prevailed (Fig. 3), the number of species of planktonic algae (74) was higher than in the Eastern Balkhash (69). Bacillariophyta primarily formed the species richness in communities in brackish eastern part. Despite the specified distinctions, trends of distribution of the abundance of species per taxonomic Division almost coincided in eastern and western parts of the water area, which testified to the similarity of species structure in the lake as a whole. In the Western Balkhash, dinophyte Peridinium sp., diatom Cyclotella meneghiniana, euglenophyte Trachelomonas sp. were found rather often. In the Eastern Balkhash, besides the two latter species, diatom Navicula sp., green Franceia sp., cyanobacteria Snowella lacustris, Gomphosphaeria aponina, and Gloeocapsa sp. were widespread.

In relation to the habitat preferences, planktonic-benthic and planktonic species were prevalent within phytoplankton. Revealed species-indicators prefer alkaline waters (alkaliphiles) or are indifferent to $\mathrm{pH}$, and serve as indicators of moderately oxygenated waters, temperate temperatures, and are indifferent to salinity (Fig. 3). In comparison with the western part, the Eastern Balkhash had more planktonic algae species that are associated with temperate temperature waters, not cold, more indicators of oxygenated waters, and halophiles with presence of mesohalobes. Despite these differences, Figure 3 illustrates that the lines of polynomial trends nearly coincided for all the indicator distributions in the Eastern and Western Balkhash, except $\mathrm{pH}$-indicators. Here, there is an essential difference: the water of the eastern part of the lake, according to bioindication, is more alkaline than in the western part, and tops of trend lines are shifted into opposite directions of $\mathrm{pH}$-spectrum.

Distribution of algae species-indicators of organic pollution of water show that the water quality of the lake generally corresponded to Class III (Fig. 4) according to Sládeček (1973), or Watanabe et al. (1986). In comparison with the west, the eastern part of the lake was characterized by slightly higher level of trophy, and that has been 
reflected by the presence of saprophiles (according to Watanabe et al., 1986) within phytoplankton species. Among algae-indicators of the nutrition type, there were mostly species that use photosynthesis and are stable against moderate concentration of organic nitrogen (ate). The number of species of this ecological group of algae was higher in the Eastern Balkhash. It should be noted that in the context of difference in speciesindicators structure (Fig. 3), the distributions trends of the nutrition type indicators in the Eastern and Western Balkhash almost coincided (Fig. 4).
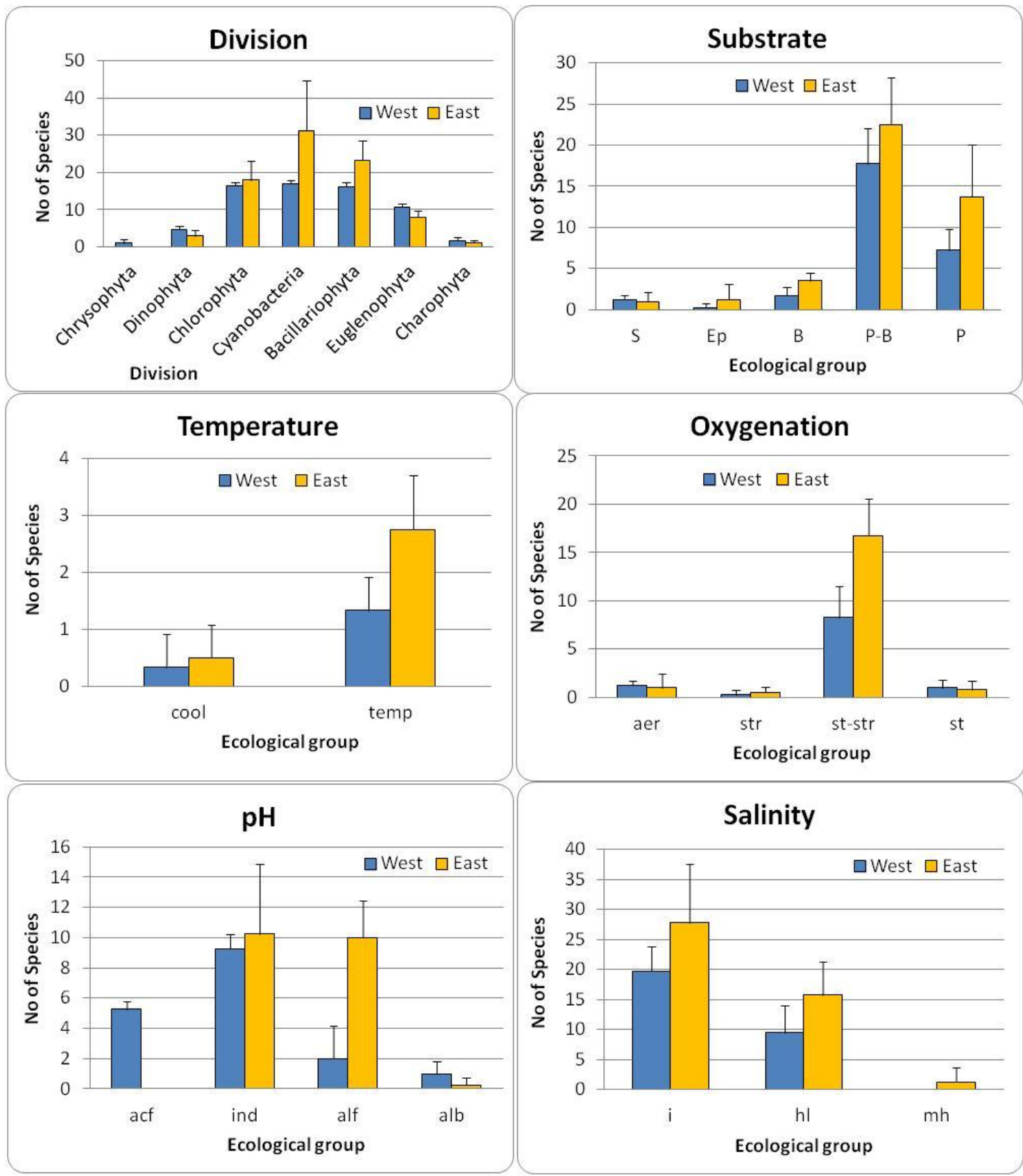

Figure 3. Distribution of the number of species-indicators over taxonomic divisions and ecological groups in the Western and Eastern Balkhash according to phytoplankton species' preferences to habitat type, water temperature, $\mathrm{pH}$, water mobility and saturation by oxygen, water $\mathrm{pH}$, and salinity in summer 2004 

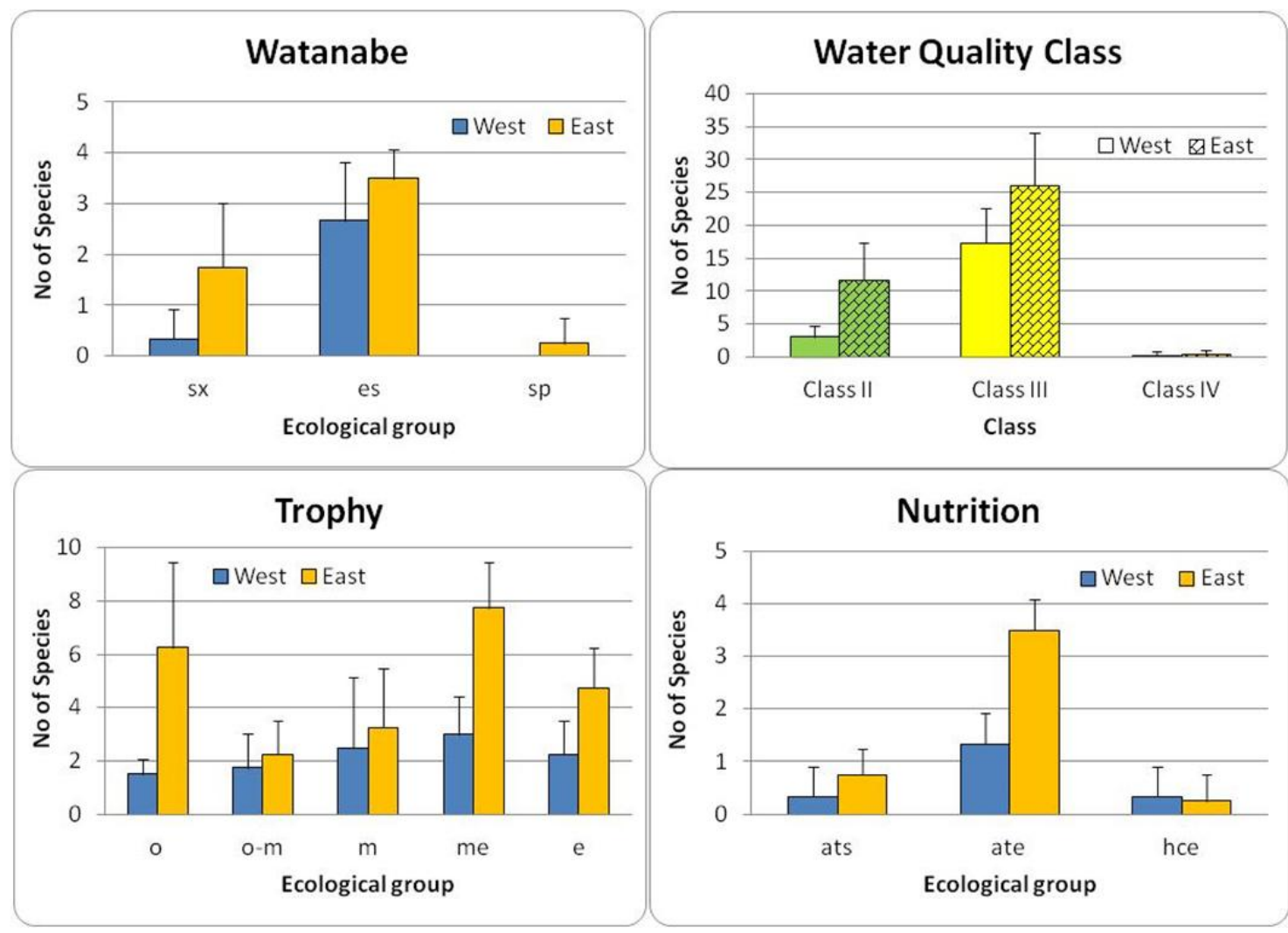

Figure 4. Assessment of the ecological state of the Balkhash Lake according to phytoplankton species-indicators in relation to organic pollution according to Watanabe (1986) and Sládeček (1973), trophic status and nutrition type, summer 2004. Coloring of the histogram in the figure of water quality classes corresponds to the codes of the EU scale: Class II - green; Class IIIyellow; Class IV-orange. Abbreviations for indicator groups of algae as in Table 3

Wide groups of algae ranging from oligotrophs to eutrophs (Fig. 4) represented indicators of trophy. In Eastern Balkhash, mesotrophs had larger number of species, and the presence of eutrophs shifted the trend line of distribution of the indicators for this part of the water area towards a eutrophy.

\section{Spatial distribution}

The set of ecological maps have been constructed to assess the spatial distribution of indicator species across the water area of the Balkhash Lake. The majority of planktonic algae species has been spread unevenly, with outbursts of species richness on limited areas. Bacillariophyta was prevail in species number in areas of the Ili River discharge (the Western Balkhash), as well as the Karatal, Lepsy and Aksu rivers (the Eastern Balkhash) (Fig. 5a).

Chrysophyta was found in the Western Balkhash only, and together with Charophyta, it was most represented in area of the Ili River discharge (Fig. 5b). The Cyanobacteria was discovered with high species richness in the eastern part of the water area $(F i g .5 c)$. The spatial distribution of Dinophyta (Fig. 5d), Euglenophyta (Fig. 5e) and Chlorophyta species richness (Fig. 5f) did not follow certain patterns, but there were more of them in the western part. 

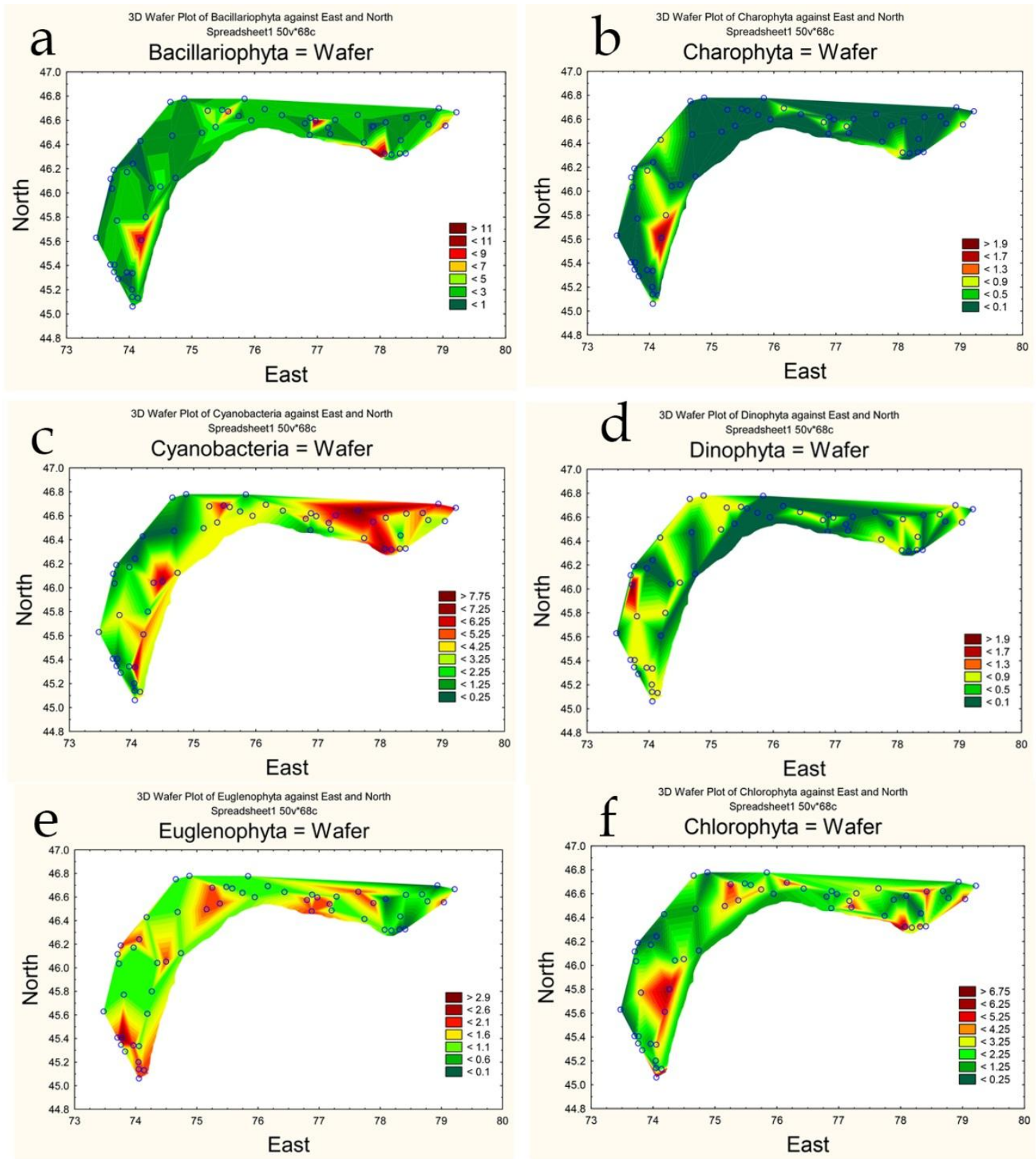

Figure 5. The distribution of total phytoplankton species richness in the algal Divisions over the water surface of the Balkhash Lake, summer 2004

We analyzed distribution of species indicators of substrate preferences. Planktonic algae prevailed in communities of the eastern deep-water part of the lake (Fig. 6a). Planktonic-benthic algae were widespread across the entire water area, with the highest species richness being recorded near the southeastern coast of the Western Balkhash and with the homogenous distribution of the number of species in the eastern part of the water area (Fig. 6b). Benthic species of algae preferred the areas of river discharge (Fig. 6c). Sparse species that have vital connection with a substrate such as epiphytes and soil related species were found across the whole lake water area with higher concentration near the coastal zones (Fig. 6d). 

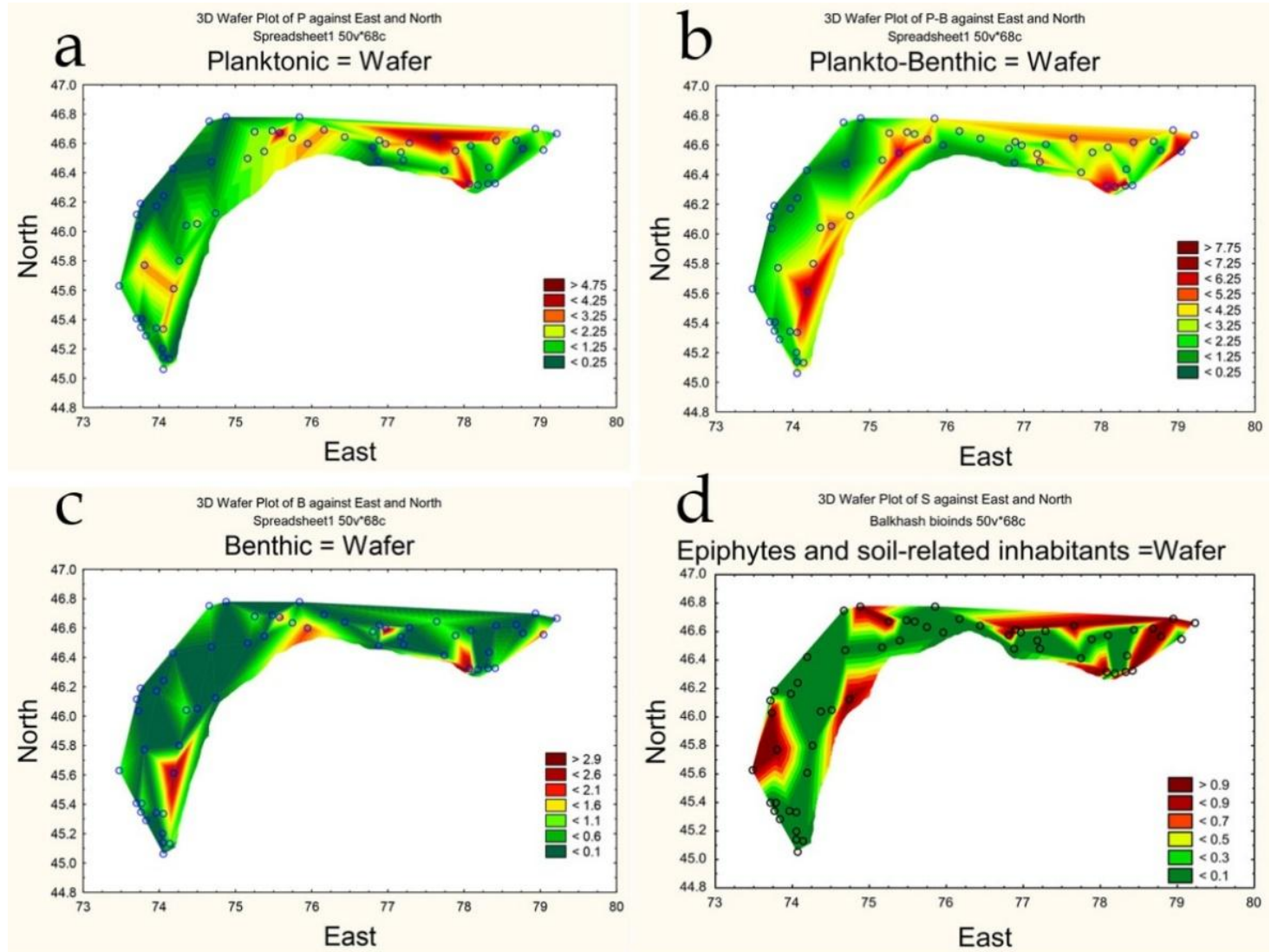

Figure 6. The distribution of algal species-indicators of habitat over the water surface of the Balkhash Lake, summer 2004

Species-indicators of moderate temperature conditions prefer water areas near the river discharge as well as shallow waters (Fig. 7a). Only few indicators of cold waters were found on some areas of water surface (Fig. $7 b)$.
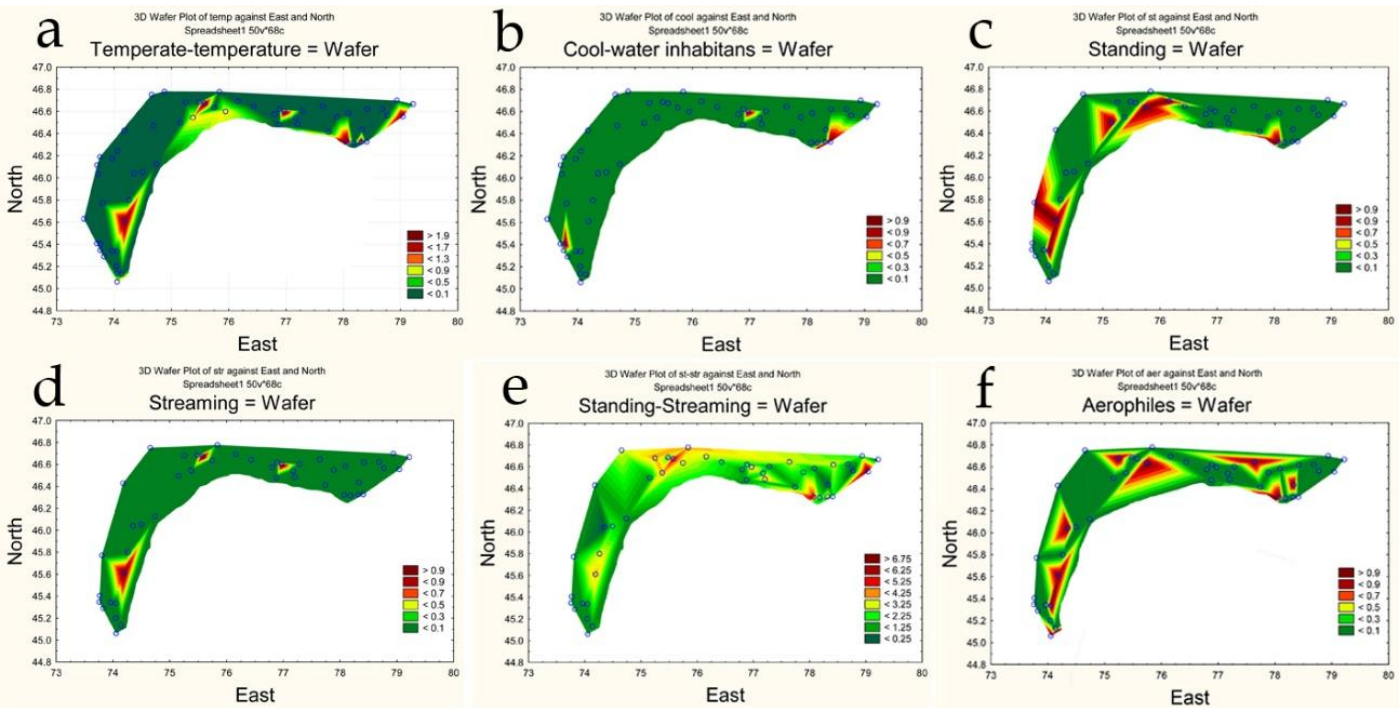

Figure 7. The distribution of algal species-indicators of the temperature and water oxygenation over the water surface of the Balkhash Lake, summer 2004 
Algae - inhabitants of the poorly oxygenated standing waters were represented by the greatest number of species in the western relatively shallow part of the lake (Fig. $7 c$ ), whereas the distribution of algae that prefer moving and oxygenated waters did not adhere to clear patterns (Fig. $7 d, e, f$ ).

Distribution maps of water $\mathrm{pH}$ indicators show that acidophiles (Fig. 8a) and alkalibionts (Fig. 8c) preferred the Western Balkhash, whereas alkaliphiles - the Eastern Balkhash (Fig. 8d). Species-indifferents were found across the whole water area, and they were drawn towards the southeastern coast of the Western Balkhash, towards northern and partially the southern and eastern coast of the Eastern Balkhash (Fig. $8 b$ ). There is notable absence of extreme groups of $\mathrm{pH}$-indicators (acidophiles and alkalibionts) in the eastern part of the lake. That is, the western part of the water area is more varied regarding of water $\mathrm{pH}$. Apparently, it is connected with the inflow of fresh water from the Ili River, and also with the general heterogeneity of conditions in the Western Balkhash.
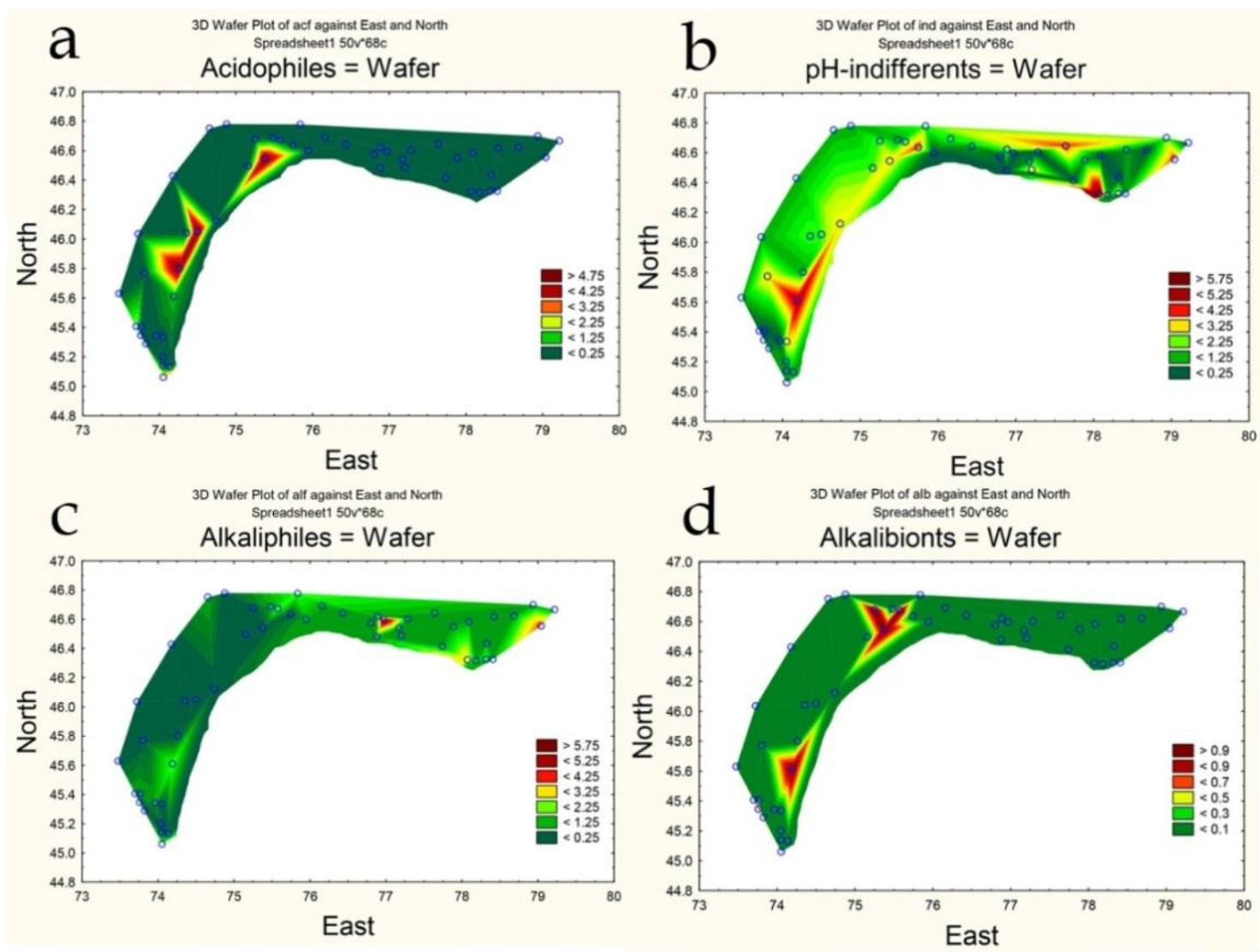

Figure 8. The distribution of algal species-indicators of water $\mathrm{pH}$ over the water surface of the Balkhash Lake, summer 2004

Maps of salinity indicators distribution demonstrated that mesohalobes were revealed only in the east brackish part of the water area (Fig. 9a). Species salinity-indifferents were widespread across the whole water area (Fig. 9c), and halophiles preferred the Eastern Balkhash and adjacent part of the water area of the Western Balkhash (Fig. 9b).

Algae - indicators of trophic state ranged from oligotraphentic to eutraphentic. Oligotraphentic species were most various in the Eastern Balkhash (Fig. 10a), oligo- 
mesotraphentic - in the Western Balkhash (Fig. 10b). Mesotraphentic, mesoeutraphentic and eutraphentic algae were recorded across the whole water area (Fig. $10 c, d, e)$.
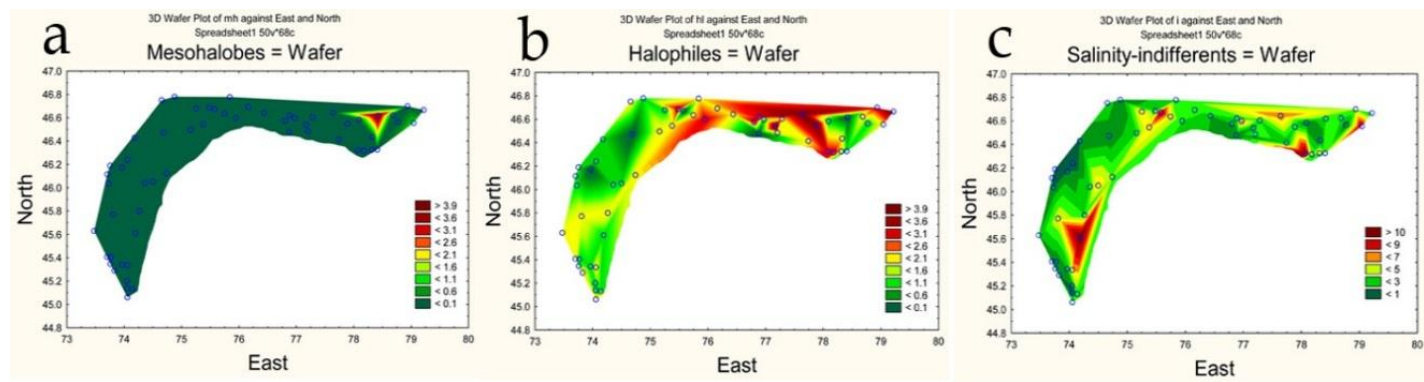

Figure 9. The distribution of algae species-indicators of salinity over the water surface of the Balkhash Lake, summer 2004
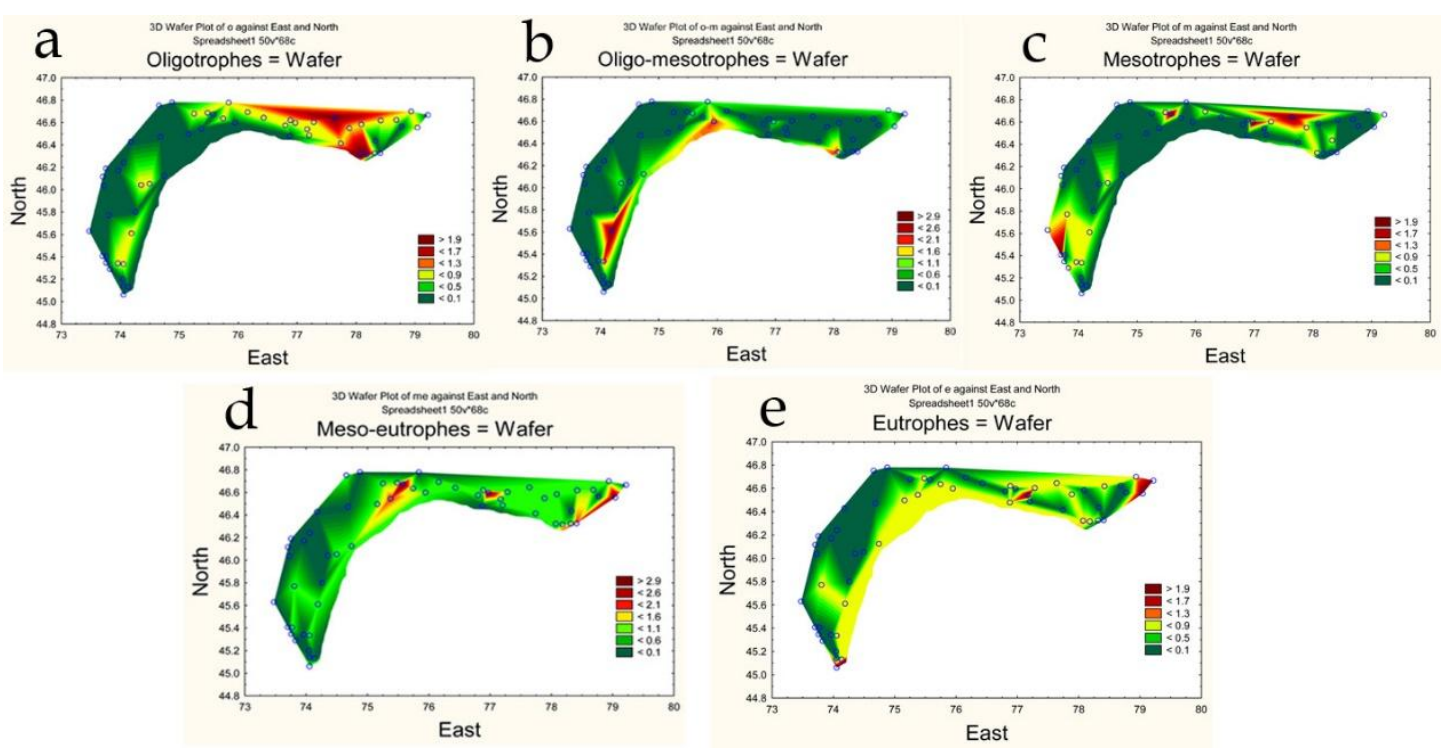

Figure 10. The distribution of algae species - trophic indicators over the water surface of the Balkhash Lake, summer 2004

The maps of distribution of species-indicators of nitrogen uptake metabolism show that nitrogen-autotrophic taxa, tolerating very small concentrations of organically bound nitrogen (Fig. 11a) and nitrogen-autotrophic taxa, tolerating elevated concentrations of organically bound nitrogen (Fig. $11 \mathrm{~b}$ ) preferred areas of river drain discharge and shallow areas of the Eastern Balkhash. Trace amounts of facultative heterotrophs were found across the whole water area of the lake (Fig. 11 c). Indicators of organic pollution (according to Watanabe, 1986) demonstrated similar distribution patterns for inhabitants of pure waters - saproxenes (Fig. 11d) and those of waters moderately saturated with organic matter - eurysaprobes (Fig. 11e).

The majority of algal indicators of organic pollution according to Sládeček (1973) pointed to the moderate level that corresponds to water quality class III (Fig. 12). Average values of saprobity index S (Table 2) confirmed this conclusion, even though they were slightly higher in the western part of the lake. 

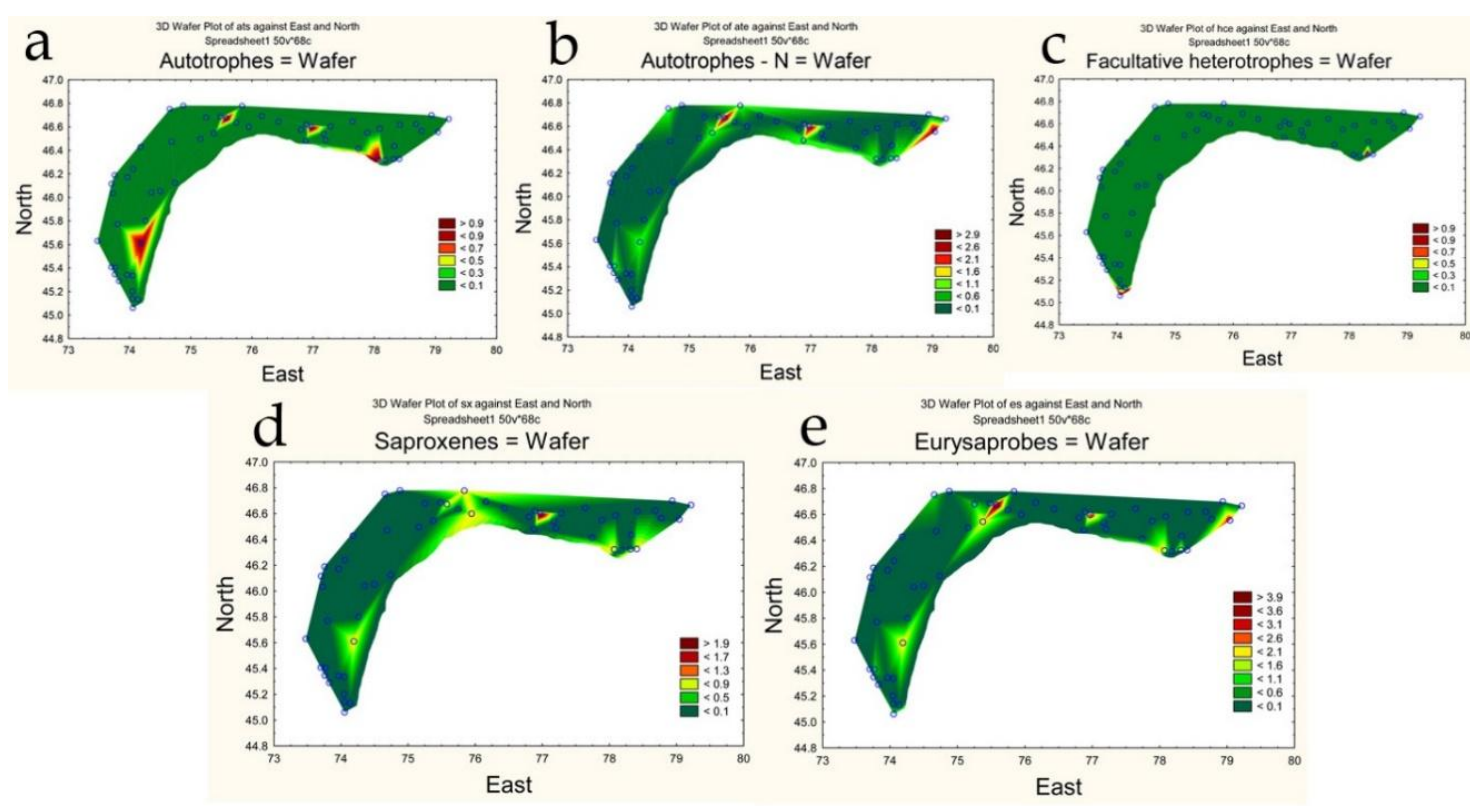

Figure 11. The distribution of algal species - nutrition type indicators over the water surface of the Balkhash Lake, summer 2004
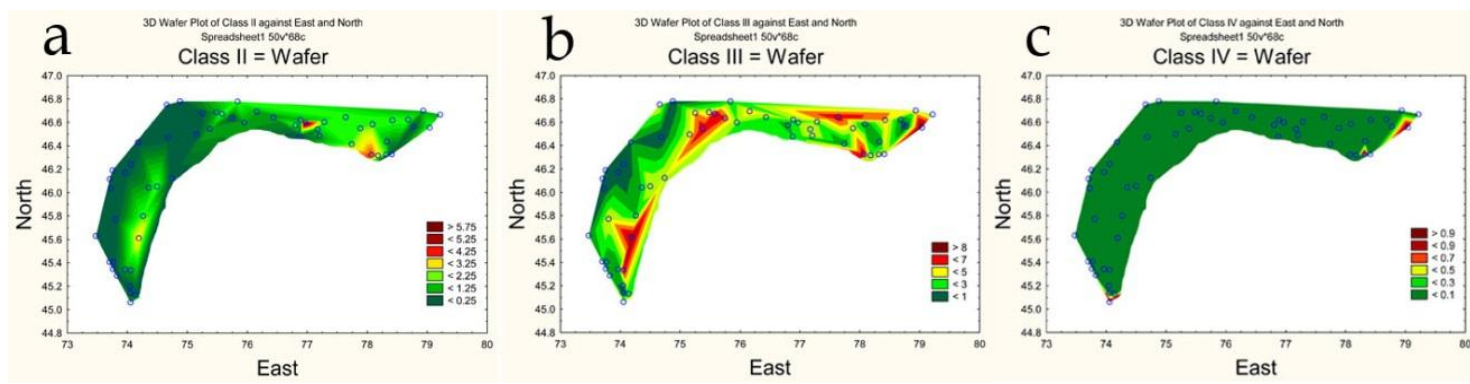

Figure 12. The distribution of algal species-indicators of the water quality class over the water surface of the Balkhash Lake, summer 2004

No significant correlation was revealed in the distribution of indicator species of phytoplankton and the content of heavy metals in water of the Balkhash Lake. The influence of the total concentration of toxicants on phytoplankton was evident using WESI index. The considerable part of the water area of the Western Balkhash was characterized by the WESI index being less than one, and that demonstrated an oppression of photosynthetic activity of algae (Fig. 13). According to index values, the toxic pollution did not affect the phytoplankton communities in the Eastern Balkhash.

\section{Discussion}

The Balkhash Lake is a unique natural water body with gradient of salt saturation of water along a longitudinal axis of the lake. Besides hydrochemical conditions, the western and eastern parts of Balkhash differ in other environmental variables. As can be seen on summer 2004 investigation results, water of the Western Balkhash contained increased concentrations of nitrates, nitrites, total phosphorus, iron, zinc and copper, 
and in the Eastern Balkhash, phosphates, easily oxidized organic matter, cadmium, lead, nickel and cobalt were prevalent.

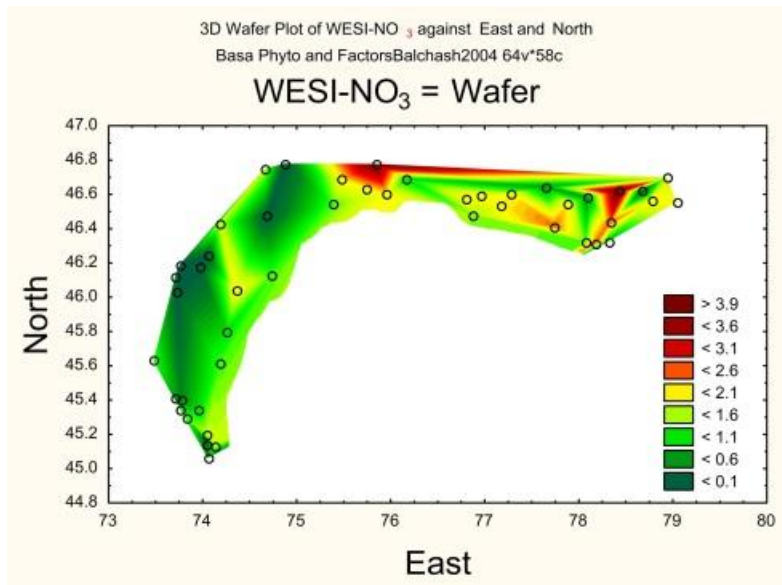

Figure 13. The distribution of the WESI index values over the water surface of the Balkhash Lake, summer 2004

Phytoplankton in the Balkhash Lake was represented by 92 species of algae in summer 2004. Bacillariophyta prevailed in species richness in brackish eastern part, whereas Chlorophyta in the Western Balkhash. Differences between total number of algal species in western freshwater part (74) and eastern brackish part of Balkhash (69) were insignificant. In the same time, a negative correlation was found between the number of species in phytoplankton community and water salinity in water body of another regions such as Eastern Mediterranean (Barinova et al., 2009), Northern (Klymiuk et al., 2014; Barinova et al., 2015b) and Eastern Black Sea region (Bilous et al., 2016), and Northern Kazakhstan (Barinova et al., 2011). Mapping illustrated that local outbreaks of the species richness Bacillariophyta, partly Charophyta, Cyanobacteria, Chlorophyta are associated with the shallow, macrophytic areas of the water surface that are under the influence of river discharge. We have previously demonstrated a positive relationship between the spatial distribution of the total number of species of plankton algae along the Balkhash Lake and macrophytes coverage (Barinova et al., 2017). Detailing the results obtained earlier in the present study showed that the species of the Bacillariophyta, Charophyta, Cyanobacteria, and Chlorophyta divisions contribute roughly to the increase in species richness in the areas where macrophytes are developed.

The analysis of published data showed that the relationship between the species richness of hydrocenosis and macrophytes could be both positive and negative (Peretyatko et al., 2009; Muylaert et al., 2010; Pieczyńska et al., 1999; KuczyńskaKippen and Joniak, 2015). Previously noted positive relationship between macrophytes and the number of species of phytoplankton in the water bodies in the arid zone of Kazakhstan (Barinova and Krupa, 2017) may have been because the presence of macrophytes enhances the role of small species in zooplankton, mainly rotifers (Krupa, 2012), that has no significant effect on algae.

The data visualization showed that the planktonic species are more widely represented in the Eastern Balkhash, which is consistent with the increase in depth in this part of the water area. The active reaction of water clearly divided the 
phytoplankton communities into two parts: of the Western Balkhash with predominance of $\mathrm{pH}$-indifferent species and of the Eastern Balkhash with predominance of alkaliphiles, which is coherent with our $\mathrm{pH}$ measurements. The predominance of mesohalobes and halophiles in the phytoplankton of the Eastern Balkhash reflected a higher concentration of dissolved salts in this part of the lake water. The response of phytoplankton to the temperature and water oxygen saturation was approximately the same for the Eastern and Western Balkhash, while the zones of influence of river discharge and shallow waters substantially affected the distribution of indicator species.

Bioindication data has identified the Balkhash Lake as moderately polluted, with water quality class III. The distribution of algal species - indicators of the trophic state (the presence of nutrients) and the ratio of species to the total level of organic pollution of water (according to Sládeček) made for different estimates of the eastern and western parts of the lake. In comparison with the west, the eastern part of Balkhash has been characterized by a higher level of trophy, as evidenced by the appearance of saprophiles in the phytoplankton composition, the presence of more eutrophic species and species resistant to moderate concentrations of organic nitrogen.

This evaluation was supported by the spatial dynamics of phosphates (Table 2) which are present in low concentrations in the reservoirs of Kazakhstan (Nikolaenko, 1989) and therefore have a stronger limiting effect on phytoplankton compared to nitrogen compounds. Maps of the spatial distribution of algal species - trophic indicators showed a higher content of nutrients in the mouths of rivers and in shallow waters. The water quality assessment by Sládeček (1973) also characterized a level of organic pollution of the Balkhash Lake as moderate and mesosaprobic, however, with slightly higher values of the saprobity index in the western part. This assessment showed that pollutants primarily come with the waters of the Ili River which flows into the Western Balkhash. This confirms the conclusion we made earlier when analyzing the spatial distribution of biogenic elements and total phosphorus in the waters of the Balkhash Lake (Krupa et al., 2017b). The supply of nutrient elements with river waters stimulated an increase in the quantitative variables of phytoplankton communities in this zone, which we showed earlier (Krupa et al., 2018).

The effect of total toxic pollution on phytoplankton of the Balkhash Lake has not been tracked when analyzing the distribution of indicator species, but it was evident when using the WESI index. The small values of the index -0.46 on average, have been recorded in the zone of the Ili River discharge (Fig. 1) and on the opposite coast of the lake -0.97 on average, where the discharge current flows directly from the estuarine zone. The main contribution to the inhibition of algal communities in this part of the western Balkhash water area was by zinc and copper that come with river waters at concentrations of $0.080-0.264 \mathrm{mg} \mathrm{dm}^{-3}$ and $0.02-0.06 \mathrm{mg} \mathrm{dm}^{-3}$. Thus, the pollutants entering the lake along with the river runoff have a mutually opposite effect on the communities of Lake Balkhash. Biogenic elements have a stimulating effect on phytoplankton, whereas an increased level of toxic pollution partially suppresses its development. This was only possible to reveal using the WESI index.

The average content of zinc and copper was also higher in the western part of the water area compared to the eastern. In the Eastern Balkhash, there was cadmium, lead, nickel and cobalt; however, the total amount of toxic substances did not adversely affect the phytoplankton of this part of the lake, as evidenced by the WESI index values. That means high self-purification capacity of the Balkhash Lake ecosystem. This may be due to the increased content of dissolved salts in the Eastern Balkhash, which together with 
high $\mathrm{pH}$-values (Table 1) weaken the toxic properties of heavy metals by making the latter insoluble (Nikanorov and Zhulidov, 1991). The stress that we have identified for algal communities in the Western Balkhash is not only related to anthropogenic influence, but is also caused by natural increased potentially toxic elements background due to the lake being located in the metallogenic province (Mazurov, 2005). Earlier, we have detected an increased content of some heavy metals and their negative impact on algal communities of small lakes in Kazakhstan, both in protected areas (Krupa et al., 2016; Krupa and Barinova, 2016) and in conditions of moderate anthropogenic pollution (Barinova et al., 2011). That said the alkaline reaction of the lake water partially neutralizes the toxic effect of heavy metals on phytoplankton as evidenced in the eastern part of the Balkhash Lake.

\section{Conclusion}

In summer 2004, phytoplankton of the Balkhash Lake was represented by 92 species. In the western, freshwater part of the lake, green algae prevailed, in the eastern, brackish part it was diatoms. The larger total number of algal species in the Western Balkhash (74), compared to the Eastern one (69). Mapping showed a positive relationship between the species richness of algal Divisions and macrophytes. Changes of the proportion of indicator species within the phytoplankton composition reflected the spatial gradient of environmental variables - an increase in depth, TDS and $\mathrm{pH}$-values in the direction from west to east. According to bioindication analysis, the water of the Balkhash Lake is moderately polluted, water quality class III with high self-purification of its ecosystem. The WESI index calculation revealed the effect of the total toxic pollution on the phytoplankton of the Western Balkhash. The spatial distribution of the WESI index showed that the pollutants entering the lake with river flow had a mutually opposite effect on the phytoplankton communities: the stimulating effect of biogenic elements from one hand, and the partial suppression by an increased level of toxic pollution from the other.

In general, the method of statistical mapping of indicators in combination with bioindication based on the algae can be recommended for use of future monitoring of changes in water quality and identify sources of pollution of the unique natural water body - the Balkhash Lake.

Acknowledgements. The work was partly supported by Committee of Science, Ministry of Education and Science, Republic of Kazakhstan as well as by the Israeli Ministry of Absorption.

\section{REFERENCES}

[1] Abrosov, V. N. (1973): Lake Balkhash. - Science, Leningrad (in Russian).

[2] Barinova, S. (2011): Algal Diversity Dynamics, Ecological Assessment, and Monitoring in the River Ecosystems of the Eastern Mediterranean. - Nova Science Publishers, New York.

[3] Barinova, S. (2017a): On the classification of water quality from an ecological point of view. - International Journal of Environmental Sciences \& Natural Resources 2(2): 1-8. DOI: 10.19080/IJESNR.2017.02.555581.

[4] Barinova, S. S. (2017b): Empirical model of the functioning of aquatic ecosystems. International Journal of Oceanography and Aquaculture 1(3): 1-9. 
[5] Barinova, S., Chekryzheva, T. (2014): Phytoplankton dynamic and bioindication in the Kondopoga Bay, Lake Onego (Northern Russia). - J. Limnol 73(2): 282-297. DOI: 10.4081/jlimnol.2014.820. http://dx.doi.org/10.4081/jlimnol.2014.820.

[6] Barinova, S., Fahima, T. (2017): The Development of the a World Database of Freshwater Algae-Indicators. - Journal of Environment and Ecology 8(1): 1-7. http://www.macrothink.org/journal/index.php/jee/article/view/11228/8981.

[7] Barinova, S., Krupa, E. (2017): Bioindication of ecological state and water quality by phytoplankton in the Shardara Reservoir, Kazakhstan. - Environment and Ecology Research 5: 73-92. DOI: 10.13189/eer.2017.050201.

[8] Barinova, S. S., Medvedeva, L. A., Anissimova, O. V. (2006): Diversity of Algal Indicators in Environmental Assessment. - Pilies Studio, Tel Aviv (in Russian).

[9] Barinova, S. S., Bragina, T. M., Nevo, E. (2009): Algal species diversity of arid region lakes in Kazakhstan and Israel. - Community Ecology 10(1): 7-16. DOI: 10.1556/ComEc.10.2009.1.2.

[10] Barinova, S. S., Nevo, E., Bragina, T. M. (2011): Ecological assessment of wetland ecosystems of northern Kazakhstan on the basis of hydrochemistry and algal biodiversity. - Acta Botanica Croatica 70(2): 215-244. DOI: 10.2478/v10184-010-0020-7.

[11] Barinova, S. S., Klochenko, P. D., Belous, Y. P. (2015a): Algae as indicators of the ecological state of water bodies: methods and prospects. - Hydrobiological Journal 51(6): 3-21.

[12] Barinova, S., Klymiuk, V., Lyalyuk, N. (2015b): Ecology of phytoplankton in the regional landscape park "Slavyansky Resort", Ukraine. - Applied Ecology and Environmental Research 13(2): 449-464. DOI: 10.15666/aeer/1302_449464.

[13] Barinova, S., Bilous, O., Ivanova, N. (2016): New statistical approach to spatial analysis of ecosystem of the Sasyk Reservoir, Ukraine. - International Journal of Ecotoxicology and Ecobiology 1(3): 118-126. DOI: 10.11648/j.ijee.20160103.19.

[14] Barinova, S., Krupa, E., Kadyrova, U. (2017): Spatial dynamics of species richness of phytoplankton of Lake Balkhash (Kazakhstan) in the gradient of abiotic factors. Transylv. Rev. Syst. Ecol. Res. "The Wetlands Diversity" 19(2): 1-18.

[15] Bilous, O. P., Barinova, S. S., Ivanova, N. O. and Huliaieva, O. A. (2016): The use of phytoplankton as an indicator of internal hydrodynamics of a large seaside reservoir-case of the Sasyk Reservoir, Ukraine. - Ecohydrol. Hydrobiol. 16: 160-174.

[16] Fokina, A. S. (1982): The Present State of Phytoplankton in the Balkhash Lake. - In: Gvozdev, E. (ed.) Forecast of the Integrated and Rational Use of Natural Resources, Their Protection and Prospects for the Development of The Productive Forces of the Lake Basin. Balkhash in the Period until 1990-2000. Part 2. Science of KazSSR, Alma-Ata (in Russian).

[17] Fomin, G. S. (1995): Water. Control of Chemical, Bacterial and Radiation Safety according to International Standards. - NGO “Alternative”, Moscow (in Russian).

[18] Fritioff $\mathrm{A}^{\circ}$., Kautsky, L., Greger, M. (2005): Influence of temperature and salinity on heavy metal uptake by submersed plants. - Environmental Pollution 133: 265-274.

[19] Gollerbach, M. M., Kossinskaya, E. K., Polyansky, V. I. (1953): Key to Freshwater Algae of USSR. Vol. 2. Blue-Green Algae. - Soviet Science, Moscow (in Russian).

[20] Heywood, V. (2004): Modern approaches to floristics and their impact on the region of SW Asia. - Turk. J. Bot. 28: 7-16.

[21] Hustedt, F. (1938-1939): Systematische und Ökologische Untersuchungen über die Diatomeenflora von Java, Bali und Sumatra. - Arch. Hydrobiol. Suppl. 15: 131-177, 393-506, 638-790; 16, 1-155, 274-394.

[22] Hustedt, F. (1957): Die Diatomeenflora des Flußsystems der Weser im Gebiet der Hansestadt Bremen. - Abhandl. Naturwis Ver. Bremen 34: 181-440.

[23] Kiselev, I. A. (1956): Methods of study of plankton. Life of Freshwaters of the USSR. Vol. 4. - USSR Academy of Sciences, Moscow, Leningrad (in Russian). 
[24] Klymiuk, V., Barinova, S., Lyalyuk, N. (2014): Diversity and ecology of algal communities from the regional landscape park "Slavyansky Resort", Ukraine. - Research and Reviews. Journal of Botanical Science 3(2): 9-26.

[25] Klymiuk, V., Barinova, S. (2016): Phytoplankton cell size in saline lakes. - Research Journal of Pharmaceutical, Biological and Chemical Sciences 7(1): 1077-1085.

[26] Krupa, E. G. (2012): Zooplankton of Limnic and Lotic Ecosystems of Kazakhstan. Structure, Patterns of Formation. - Palmarium Academic Publishing, Saarbrucken (in Russian).

[27] Krupa, E. G., Barinova, S. S. (2016): Environmental variables regulating the phytoplankton structure in high mountain lakes. - Research Journal of Pharmaceutical, Biological and Chemical Sciences 7(4): 1251-1261.

[28] Krupa, E. G., Tsoi, V.N, Lopareva, T.Ya., Ponomareva, L. P., Anurieva, A. N., Sadyrbaeva, N. N., Asylbekova, S.Zh., Isbekov, K. B. (2013): Long-term dynamics of the hydrobionts of Lake Balkhash and its relation to environmental factors. - Bulletin of the Astrakhan State Technical University. Series: Fisheries (2): 85-96 (in Russian).

[29] Krupa, E. G., Sadyrbaeva, N. N., Ponomareva, L. P., Anurieva, A. N., Assylbekova, S.Zh. (2014a): The long-term variability of phytoplankton, zooplankton and macrozoobenthos in the Lake Balkhash depending on global climatic factors. - Bulletin of KazNU, Ecological Series 1(40): 296-304 (in Russian).

[30] Krupa, E., Slyvinskiy, G., Barinova, S. (2014b): The effect of climatic factors on the long-term dynamics of aquatic ecosystem of the Balkhash Lake (Kazakhstan, Central Asia). - Advanced Studies in Biology 6(3): 115-136.

[31] Krupa, E. G., Barinova, S. M., Romanova, S. M., MalybekoV, A. B. (2016): Hydrobiological assessment of the high mountain Kolsay Lakes (Kungey Alatau, Southeastern Kazakhstan) ecosystems in climatic gradient. - British Journal of Environment and Climate Change 6(4): 259-278.

[32] Krupa, E. G., Barinova, S. S., Tsoy, V. N., Sadyrbaeva, N. N. (2017a): Formation of phytoplankton of Lake Balkhash (Kazakhstan) under the influence of major regional climatic factors. - Advances in Biology \& Earth Sciences 2(2): 204-213 (in Russian).

[33] Krupa, E. G., Barinova, S. S., Tsoy, V. N., Lopareva, T. Y., Sadyrbaeva, N. N. (2017b): Spatial analysis of hydrochemical and toxicological variables of the Balkhash Lake, Kazakhstan. - Research Journal of Pharmaceutical, Biological and Chemical Sciences 8(3): $1827-1839$.

[34] Krupa, E. G., Barinova, S.S, Ponamareva, L., Tsoy, V. N. (2018): Statistical mapping and 3-D surface plots in phytoplankton analysis of the Balkhash Lake (Kazakhstan). Transylv. Rev. Syst. Ecol. Res. "The Wetlands Diversity” 20(1): 1-16.

[35] Kuczyńska-Kippen, N., Joniak, T. (2015): Zooplankton diversity and macrophyte biometry in shallow water bodies of various trophic state. - Hydrobiologia 774(1): 39-51. DOI: $10.1007 / \mathrm{s} 10750-015-2595-4$.

[36] Kudekov, T. K. (Ed.) (2002): The Modern Ecological State of the Basin of Lake Balkhash. - Kaganat, Almaty (in Russian).

[37] Leonov, M. G. (Ed.) (2013): Lateral Tectonic Flows in the Lithosphere of the Earth. GEOS, Moscow (in Russian).

[38] Li, H., Shi, A., Li, M., Zhang, X. (2013): Effect of pH, temperature, dissolved oxygen, and flow rate of overlying water on heavy metals release from storm sewer sediments. Hindawi Journal of Chemistry 2013, Article ID 434012. http://dx.doi.org/10.1155/2013/434012.

[39] Matvienko, A. M. (1954): Key to Freshwater Algae USSR. Vol. 3. Golden Algae. Soviet Science, Moscow (in Russian).

[40] Mazurov, A. K. (2005): Metallogenic zoning of Kazakhstan. - Proceedings of Tomsk Polytechnic University 308(4): 33-39 (in Russian).

[41] Moshkova, N. A., Gollerbach, M. M. (1986): Key to Freshwater Algae USSR. Vol. 10(1). Green Algae. Class Ulothrichophyceae. - Soviet Science, Moscow (in Russian). 
[42] Muylaert, K., Pérez-Martinez, C., Sánchez-Castillo, P., Lauridsen, T. L., Vanderstukken, M., Declerck, S. A. J., Van Der Gucht, K., Conde-Porcuna, J.-M., Jeppesen, E., De Meester, L., Vyverman, W. (2010): Influence of nutrients, submerged macrophytes and zooplankton grazing on phytoplankton biomass and diversity along a latitudinal gradient in Europe. - Hydrobiologia 653: 79-90. DOI 10.1007/s10750-010-0345-1.

[43] Nikanorov, A. M., Zhulidov, A. V. (1991): Biomonitoring of Metals in Freshwater Ecosystems. - Gidrometeoizdat, Leningrad (in Russian).

[44] Nikolaenko, V. A. (1989): Hydrochemical regime and water quality of the reservoirs of Central Asia. - Hydrobiological Journal 25(1): 78-83 (in Russian).

[45] Palamar-Mordvintseva, G. M. (1982): Key to Freshwater Algae USSR. Vol. 11(2). Green Algae. Class Conjugatophyceae. Desmidiaceae (2). - Soviet Science, Moscow (in Russian).

[46] Peretyatko, A., Teissier, S., De Backer, S., Triest, L. (2009): Restoration potential of biomanipulation for eutrophic peri-urban ponds: the role of zooplankton size and submerged macrophyte cover. - Hydrobiologia 634: 125-135. DOI 10.1007/s10750-0099888-4.

[47] Pieczyńska, E., Kołodziejczyk, A., Rybak, J. I. (1999): The responses of littoral invertebrates to eutrophication-linked changes in plant communities. - Hydrobiologia 391: 9-21.

[48] Ponomareva, L. P., Shauharbaeva, D. S., Lopareva, T. Y. (2005): Hydrochemical Variables and Phytoplankton as Indicators of the Trophy State of the Balkhash Lake and Waterbodies of the Lower Delta of the Ily River. - In: Alpeisov, S. (ed.) Fisheries Research in the Republic of Kazakhstan: History and Current Status. Bastau, Almaty (in Russian).

[49] Popova, T. G. (1955): Key to Freshwater Algae USSR. Vol. 7. Euglenophyta. - Soviet Science, Moscow. (in Russian).

[50] Semenova, A. D. (Ed.) (1977): Guideline for chemical analysis of surface water. Gidrometeoizdat, Leningrad. (in Russian).

[51] Sidorenko, A. V. (Ed.) (1972): Geology of the USSR. Central Kazakhstan. T. 20. Book 1. - Nedra, Moscow. (in Russian).

[52] Sládeček, V. (1973): System of water quality from the biological point of view. Ergebnisse der Limnologie 7: 1-128.

[53] Tarasov, M. N. (1961): Hydrochemistry of Balkhash Lake. - Academy of Sciences of the USSR, Moscow. (in Russian).

[54] Van Dam, H., Martens, A., Sinkeldam, J. (1994): A coded checklist and ecological indicator values of freshwater diatoms from the Netherlands. - Netherlands J. Aquatic Ecol 28(1): 117-133.

[55] Vorobyeva, N. B., Tyutenkov, S. K., Sadukasova, R. E., Fokina, A. S. (1982): The Current State and Prospects for the Development of the Fodder Base for Fish in the Balkhash Lake Depending on the Level Regime. - In: Gvozdev, E. (ed.) Forecast of the Integrated and Rational use of Natural Resources, their Protection and Prospects for the Development of the Productive Forces of the Lake Basin. Balkhash in the Period until 1990-2000. Part 2. Science of KazSSR, Alma-Ata (in Russian).

[56] Watanabe, T., Asai, K., Houki, A. (1986): Numerical estimation to organic pollution of flowing water by using the epilithic diatom assemblage - Diatom Assemblage Index (DAIpo). - Science of Total Environment 55: 209-218.

[57] Zabelina, M. M., Kiselev, I. A., Proshkina-Lavrenko, A. I., Sheshukova, V. S. (1951): Key to Freshwater Algae USSR. Vol. 4. Diatoms. - Soviet Science, Moscow (in Russian). 